\title{
Topological anti-topological fusion in four-dimensional superconformal field theories
}

\author{
Kyriakos Papadodimas \\ Institute for Theoretical Physics, University of Amsterdam, \\ Valckenierstraat 65, 1018 XE, Amsterdam, The Netherlands \\ E-mail: k.papadodimas@uva.nl
}

ABSTRACT: We present some new exact results for general four-dimensional superconformal field theories. We derive differential equations governing the coupling constant dependence of chiral primary correlators. For $\mathcal{N}=2$ theories we show that the Zamolodchikov metric on the moduli space and the operator mixing of chiral primaries are quasi-topological quantities and constrained by holomorphy. The equations that we find are the four-dimensional analogue of the $t t^{*}$ equations in two-dimensions, discovered by the method of "topological anti-topological fusion" by Cecotti and Vafa. Our analysis relies on conformal perturbation theory and the superconformal Ward identities and does not use a topological twist.

KEYwords: Supersymmetric gauge theory, Extended Supersymmetry, Conformal Field Models in String Theory

ARXIV EPRINT: 0910.4963 


\section{Contents}

$\begin{array}{lll}1 & \text { Introduction } & 2\end{array}$

$2 \mathcal{N}=2$ superconformal field theories in four dimensions $\quad 4$

2.1 The algebra and the chiral ring 4

2.2 Superconformal Ward identities 5

$\begin{array}{lll}3 & \text { Marginal deformations and operator mixing } & 6\end{array}$

4 Marginal deformations in $\mathcal{N}=2$ superconformal field theories 9

$\begin{array}{ll}4.1 \text { Holomorphy of the chiral ring and WDVV equations } & 10\end{array}$

$\begin{array}{ll}4.2 \text { The } t t^{*} \text { equations } & 12\end{array}$

$\begin{array}{ll}4.3 \text { A flat connection } & 13\end{array}$

5 Derivation of the four-dimensional $t t^{*}$ equations $\quad 14$

6 The Zamolodchikov metric on the moduli space 17

$7 \mathcal{N}=1$ theories $\quad 18$

$8 \mathcal{N}=4$ theories $\quad 19$

$\begin{array}{ll}\text { 8.1 The moduli space for } \mathcal{N}=4 \text { gauge theories } & 19\end{array}$

$\begin{array}{ll}8.2 & \text { Exotic } \mathcal{N}=4 \text { theories? }\end{array}$

8.3 Chiral primaries for $\mathcal{N}=4$ theories 20

9 A comment on the moduli space of vacua 21

10 Discussions $\quad 22$

A Superconformal Ward identities $\quad 24$

B Some basic properties of $\mathcal{N}=2$ superconformal theories 26

$\begin{array}{ll}\text { C Contours } & 28\end{array}$

$\begin{array}{lll}\text { C.1 Scalars } & 29\end{array}$

C.2 Spin $1 \quad 31$

C.3 $\quad$ Spin $\geq 2 \quad 31$

C.4 Final answer for $\mathcal{E} \quad 31$

D Chiral-Antichiral OPE 


\section{Introduction}

Supersymmetric field theories in four dimensions are interesting for their possible phenomenological applications and as toy models for the analysis of non-perturbative phenomena in quantum field theory. Supersymmetry allows us to go beyond perturbation theory and to derive exact results, which proved important for the analysis of strongly coupled gauge theories and the discovery of dualities.

In this paper we will derive some new exact results for general four-dimensional superconformal field theories. We will mainly focus on theories with $\mathcal{N}=2$ supersymmetry, but some of our results are also true for $\mathcal{N}=1$ theories. A basic example of $\mathcal{N}=2$ SCFTs in four dimensions is the $\mathcal{N}=2 \mathrm{SU}(\mathrm{N})$ gauge theory with $N_{f}=2 N$ massless quarks in the fundamental representation $[1,2]$. Other $\mathcal{N}=2$ theories in four dimensions include [3-12]. More recently a larger class of related theories has been studied by Gaiotto [13]. They arise by compactifying the six-dimensional superconformal field theory living on $N$ M5 branes on a punctured Riemann surface. Our discussion will be general and not based on any specific theory.

Many superconformal theories in four dimensions are not isolated, but come in continuous families parametrized by coupling constants, which can be freely adjusted without breaking conformal invariance. The set of possible values for these coupling constants often has the structure of a smooth manifold that we call the moduli space $\mathcal{M}$ of the conformal field theory. This space plays the role of a "parameter space" and has to be distinguished from the moduli space of vacua, like the Coulomb or Higgs branch, of any specific conformal field theory. ${ }^{1}$ Motion along $\mathcal{M}$ is generated by perturbing the conformal field theory by marginal operators. In general there may be special points on the moduli space where the conformal field theory admits a weakly coupled Lagrangian description, but at a generic point of $\mathcal{M}$ the theory is strongly coupled.

Our main goal is to develop a method that will allow us to probe the interior of the moduli space non-perturbatively. For this we will derive a set of classical differential equations for correlators of BPS operators, when considered as functions of the coupling constants. The value of such correlators in the weak-coupling regions of the moduli space provide "boundary conditions" for these differential equations, whose solutions can then give us the correlators at all values of the coupling. This is potentially useful because it may allow us to relate weak and strong coupling results and to probe the interior of the moduli space, where no weakly coupled description is available. The BPS operators that we will study are the chiral primaries with their "chiral ring" multiplication. Additionally we want to understand how superconformal invariance constrains the Zamolodchikov metric on the moduli space.

In two-dimensional $\mathcal{N}=(2,2)$ theories many exact results are known about the correlation functions of BPS operators. By considering the topologically twisted theory it was discovered that such correlators obey differential equations with respect to the coupling constants, called the Witten-Dijkgraaf-Verlinde-Verlinde (WDVV) equations [14-16].

\footnotetext{
${ }^{1}$ For example in the case of the $\mathcal{N}=4$ super Yang-Mills the moduli space is the upper half plane parametrized by $\tau=\frac{\theta}{2 \pi}+i \frac{4 \pi}{g_{Y M}^{2}}$, modded out by the action of the $\operatorname{SL}(2, \mathrm{Z})$ duality group.
} 
These equations govern the dependence of correlators with respect to holomorphic deformations of the couplings. While useful in topological field theories, it is not easy to directly apply these equations to the physical quantum field theory since for a deformation to be consistent with unitarity it must be a real linear combination of holomorphic and antiholomorphic deformations.

In [17] a beautiful method, called "topological anti-topological fusion", was developed by Cecotti and Vafa, which allows one to consider simultaneous holomorphic and antiholomorphic deformations of correlators in two-dimensional $\mathcal{N}=(2,2)$ theories. Such correlators can be computed by gluing together a hemisphere on which the the theory is topologically twisted and one where the conjugate twisting is performed. The resulting amplitude turns out to be quasi-topological and constrained to satisfy exact differential equations called the $t t^{*}$ equations. We would like to emphasize that while the derivation was based on the topologically twisted theory, the final equations are valid even in the physical, untwisted quantum field theory.

How much of this structure survives in higher dimensional supersymmetric field theories? At first thought it appears to be difficult to generalize the arguments of [17] to four-dimensional superconformal field theories. Some important ingredients of the twodimensional story, such as the correspondence between Ramond ground states and chiral primaries via spectral flow are missing in four-dimensions. However in [18] it was shown that in the case of two-dimensional $\mathcal{N}=(2,2)$ superconformal field theories, the $t t^{*}$ equations can also be derived from the point of view of standard conformal perturbation theory in the NS sector, without relying on the topological twisting. We will show that with small modifications the same arguments can be applied to four-dimensional $\mathcal{N}=2$ superconformal field theories.

To summarize our results we find that four-dimensional $\mathcal{N}=2$ superconformal field theories have rich mathematical structure, comparable to that of $\mathcal{N}=(2,2)$ theories in two dimensions, as far their chiral ring sector is concerned. We find that the Zamolodchikov metric on the moduli space $\mathcal{M}$ of a four-dimensional $\mathcal{N}=2$ theory satisfies constraints similar to those of "special geometry".

For $\mathcal{N}=1$ and $\mathcal{N}=2$ theories we show that the 3 -point functions $C_{i j}^{k}$ of chiral primary operators vary holomorphically on $\mathcal{M}$ and satisfy the WDVV integrability equations. Similarly more general "extremal correlators" of chiral primaries vary holomorphically. Finally for $\mathcal{N}=2$ theories we find that the operator mixing for chiral primaries is characterized by the fact that such operators are sections of holomorphic vector bundles over $\mathcal{M}$ whose curvature is computed by the $t t^{*}$ equations. As we will explain in the main text, these can be considered as a partial nonlinear differential equation relating the 2- and 3-point functions of chiral primaries over the moduli space. While the 2-point functions $g_{k \bar{l}}$ are not holomorphic, they are computed as solutions of these differential equations, whose coefficients are the holomorphic functions $C_{i j}^{k}$.

In this paper we will focus on the basic derivation of the four-dimensional $t t^{*}$ equations from conformal perturbation theory. We hope to report on possible applications and on the relation to the topologically twisted theory in the future [19]. 
The plan of the paper is as follows: in section 2 we briefly summarize some basic properties of $\mathcal{N}=2$ superconformal field theories in four dimensions. In section 3 we discuss aspects of the conformal perturbation theory and the operator mixing. In section 4 we present our main results for $\mathcal{N}=2$ theories. In section 5 we describe the proof of the $t t^{*}$ equations. In section 6 we analyze the constraints for the Zamolodchikov metric on the moduli space. In section 7 we consider our results for $\mathcal{N}=1$ superconformal field theories. In section 8 we make some observations about $\mathcal{N}=4$ theories and in section 9 we make some remarks about the space of vacua of such theories. In in section 10 we have some general comments and discuss possible applications and extensions.

\section{$2 \mathcal{N}=2$ superconformal field theories in four dimensions}

In this section we review some basic facts about four-dimensional $\mathcal{N}=2$ superconformal field theories. Some useful references for the algebra and its representations are [20, 21].

\subsection{The algebra and the chiral ring}

The $\mathcal{N}=2$ superconformal algebra consists of the standard conformal generators $P_{\mu}, K_{\mu}, M_{\mu \nu}, D$, the 8 supercharges $Q_{a}^{i}, \bar{Q}_{i, \dot{a}}$ and their superconformal partners $S_{i}^{a}, \bar{S}^{i, \dot{a}}$. Additionally it contains the generators of the $\mathrm{SU}(2)_{\mathrm{R}} \times \mathrm{U}(1)_{\mathrm{R}} \mathrm{R}$-symmetry algebra. Here $a, \dot{a}$ are Lorentz spinor indices and $i$ is an $\mathrm{SU}(2)_{\mathrm{R}}$ index in the $I=\frac{1}{2}$ representation. We work in conventions where the left chiral supercharges $Q_{a}^{i}$ have $\mathrm{U}(1)_{\mathrm{R}}$ charge equal to -1 . Superconformal primary operators ${ }^{2}$ are labeled by their conformal dimension $\Delta$, the Lorentz spin $(j, \bar{j})$, the $\mathrm{SU}(2)_{\mathrm{R}}$ "isospin" $I$ and the $\mathrm{U}(1)_{\mathrm{R}}$ charge $R$.

We will be interested in chiral primary operators $\phi$ which satisfy the following conditions

$$
\left[\bar{Q}_{\dot{a}}^{i}, \phi\right]=0, \quad \Leftrightarrow \quad \bar{j}=0, I=0, \Delta=\frac{R}{2},
$$

The antichiral operators $\bar{\phi}$ have $j=0, I=0, \Delta=-\frac{R}{2}$ and are annihilated by the supercharges of left chirality $Q_{a}^{i}$. Notice that we defined the "chiral primaries" as operators which are annihilated by all supercharges of one chirality, or in other words, which are chiral with respect to both $\mathcal{N}=1$ sub-algebras of the $\mathcal{N}=2$ theory. This definition implies $I=0$.

The 2-point function of chiral primaries defines the Zamolodchikov metric for these operators

$$
g_{i \bar{j}} \equiv\left\langle\bar{\phi}_{j}(\infty) \phi_{i}(0)\right\rangle
$$

Using the $\mathrm{U}(1)_{\mathrm{R}}$ conservation it is easy to show that the operator product expansion of chiral primaries is non-singular

$$
\phi_{i}(x) \phi_{j}(0)=C_{j k}^{i} \phi_{k}(0)+\ldots
$$

and that the operator $\phi_{k}(0)$ is also chiral primary of charge $R_{k}=R_{i}+R_{j}$. In this sense the chiral primary operators form a ring under OPE multiplication, called the "chiral ring" [22].

\footnotetext{
${ }^{2}$ We call conformal primary operators those which are annihilated by the $K_{\mu}$ 's. Superconformal primary operators are those which are annihilated by all $S$ and $\bar{S}$ 's, which implies that they are also annihilated by the $K_{\mu}$ 's.
} 
The constants $C_{i j}^{k}$ are the structure constants of the ring. The chiral ring coefficients are related to the 2- and 3-point functions of chiral primaries by ${ }^{3}$

$$
\begin{aligned}
C_{i j \bar{k}} & \equiv\left\langle\bar{\phi}_{k}(\infty) \phi_{i}(1) \phi_{j}(0)\right\rangle \\
C_{i j \bar{k}} & =C_{i j}^{l} g_{l \bar{k}}
\end{aligned}
$$

\subsection{Superconformal Ward identities}

In the rest of the paper we will often use superconformal Ward identities so we briefly review them here. A basic Ward identity is that for a set of local operators $\varphi_{i}$ and a supercharge $\mathbf{Q}$ we have

$$
\sum_{k=1}^{n}\left\langle\varphi_{1}\left(x_{1}\right) \ldots\left[\mathbf{Q}, \varphi_{k}\right]\left(x_{k}\right) \ldots \varphi_{n}\left(x_{n}\right)\right\rangle=0
$$

which simply expresses the "supercharge conservation".

We will also need a somewhat less familiar identity. First we remind that if $\varphi$ is a scalar $^{4}$ conformal primary operator of dimension $\Delta$ then we define

$$
\left\langle\varphi(\infty) \varphi_{1}\left(x_{1}\right) \ldots \varphi_{n}\left(x_{n}\right)\right\rangle \equiv \lim _{x \rightarrow \infty}|x|^{2 \Delta}\left\langle\varphi(x) \varphi_{1}\left(x_{1}\right) \ldots \varphi_{n}\left(x_{n}\right)\right\rangle
$$

Now let us consider the correlator of a superconformal primary operator $\varphi$ with a number of operators $\varphi_{i}$ (not necessarily primary). According to (2.1) we have

$$
\left\langle[\mathbf{Q}, \varphi](x) \varphi_{1}\left(x_{1}\right) \ldots \varphi_{n}\left(x_{n}\right)\right\rangle+\sum_{k=1}^{n}\left\langle\varphi(x) \varphi_{1}\left(x_{1}\right) \ldots\left[\mathbf{Q}, \varphi_{k}\right]\left(x_{k}\right) \ldots \varphi_{n}\left(x_{n}\right)\right\rangle=0
$$

We multiply this relation by $|x|^{2 \Delta}$ where $\Delta$ is the dimension of $\varphi$ and take the limit $x \rightarrow \infty$. The term $|x|^{2 \Delta}\left\langle[\mathbf{Q}, \varphi](x) \varphi_{1}\left(x_{1}\right) \ldots \varphi_{n}\left(x_{n}\right)\right\rangle$ goes to zero in that limit, because $[\mathbf{Q}, \varphi](x)$ is a conformal primary ${ }^{5}$ of dimension $\Delta+\frac{1}{2}$ so this term falls off at least as fast as $\frac{1}{|x|}$ while the other terms have a finite limit according to (2.2) and we have

$$
\sum_{k=1}^{n}\left\langle\varphi(\infty) \varphi_{1}\left(x_{1}\right) \ldots\left[\mathbf{Q}, \varphi_{k}\right]\left(x_{k}\right) \ldots \varphi_{n}\left(x_{n}\right)\right\rangle=0
$$

where in this sum there is no term where the supercharge $\mathbf{Q}$ acts on $\phi(\infty)$. So when applying the superconformal Ward identity (2.1) if a superconformal primary is "hidden" at infinity then $\mathbf{Q}$ does not act on it.

Of course there is nothing special about the point at infinity, since a conformal transformation can map it to any other point. The identity (2.3) is a special case of a more general Ward identity, which holds even if all insertions in the correlator are at finite locations: for a given superconformal primary $\varphi$ in the correlator, it is always possible to

\footnotetext{
${ }^{3}$ Any three points can be mapped to $\{0,1, \infty\}$ by a conformal transformation, so the 3 -point function is fixed by conformal invariance up to an overall constant.

${ }^{4}$ For operators with spin we have to multiply with the inverse 2-point function.

${ }^{5}$ From the $\mathcal{N}=2$ algebra we have $\left[K_{\mu}, \mathbf{Q}\right] \sim \overline{\mathbf{S}}$, so since $\varphi$ is a superconformal primary we have $\left[K_{\mu}, \varphi\right]=[\overline{\mathbf{S}}, \varphi]=0$ and thus $\left[K_{\mu},[\mathbf{Q}, \varphi]\right]=0$.
} 
find a linear combination of Ward identities (for $Q$ 's and $\bar{S}$ 's), in which there are no terms involving operators of the form $[Q, \varphi]$. This is achieved by applying the Ward identity for the supercurrent multiplied by a conformal Killing spinor which vanishes at the location of $\varphi$. We presented the special case $x \rightarrow \infty$ because then the argument is quicker. The general case is explained in appendix A.

\section{Marginal deformations and operator mixing}

In $\mathcal{N}=2$ superconformal field theories exactly marginal operators preserving supersymmetry must be descendants of scalar chiral primaries of dimension $\Delta=2, \mathrm{U}(1)_{\mathrm{R}}$ charge $R=4$ and their conjugates. In the rest of the paper we denote chiral primaries of this R-charge by capital letters $\Phi_{i}$, while for chiral primaries of general charge we use the notation $\phi_{i}$.

The moduli space has the structure of a complex Kähler manifold. The marginal operators are divided into holomorphic and antiholomorphic ones as ${ }^{6}$

$$
\mathcal{O}_{i}=Q^{4} \cdot \Phi_{i}, \quad \overline{\mathcal{O}}_{j}=\bar{Q}^{4} \cdot \bar{\Phi}_{j}
$$

In superspace language the two deformations can be written as $\mathcal{N}=2 \mathrm{~F}$-terms of the form

$$
\mathcal{O}_{i}=\int d^{4} \theta \Phi_{i}, \quad \overline{\mathcal{O}}_{j}=\int d^{4} \bar{\theta} \bar{\Phi}_{j}
$$

The 2-point functions of chiral primaries and those of marginal operators

$$
\left\langle\Phi_{i}(x) \bar{\Phi}_{j}(y)\right\rangle=\frac{g_{i \bar{j}}}{|x-y|^{4}}, \quad\left\langle\mathcal{O}_{i}(x) \overline{\mathcal{O}}_{j}(y)\right\rangle=\frac{G_{i \bar{j}}}{|x-y|^{8}}
$$

can be related by using (2.1) to move the $Q$ 's from $\mathcal{O}_{i}$ onto $\overline{\mathcal{O}}_{j}$ and then using the supersymmetry algebra $\left\{Q_{a}^{i}, \bar{Q}_{j, \dot{b}}\right\}=2 \delta_{j}^{i} P_{a \dot{b}}$. With appropriate normalization of (3.1) we have

$$
\left\langle\mathcal{O}_{i}(x) \overline{\mathcal{O}}_{j}(y)\right\rangle=\nabla_{y}^{2} \nabla_{y}^{2}\left\langle\Phi_{i}(x) \bar{\Phi}_{j}(y)\right\rangle
$$

which implies $G_{i \bar{j}}=192 \cdot g_{i \bar{j}}$. The quantity $G_{i \bar{j}}$ is the Zamolodchikov metric on the moduli space $\mathcal{M}$.

Now we want to analyze how the chiral ring varies as a function of the coupling constants $\left\{\lambda^{\mu}\right\}$ parametrizing $\mathcal{M}$. At first one may think that the coupling constant dependence is simply captured by the fact that the 3-point functions of chiral primaries become moduli-dependent functions $C_{i j}^{k}(\lambda)$. However the situation is somewhat more complicated due to the operator mixing of chiral primaries with the same quantum numbers under marginal deformations.

To understand this better, let us first notice that at each point on the moduli space we can choose the basis of chiral primaries in an arbitrary way. Under a coupling constant dependent change of basis $\phi_{i}(\lambda) \rightarrow U_{i}^{i^{\prime}}(\lambda) \phi_{i^{\prime}}(\lambda)$ the 3-point functions transform as $C_{i j}^{k}(\lambda) \rightarrow U_{i}^{i^{\prime}}(\lambda) U_{j}^{j^{\prime}}(\lambda) U_{k^{\prime}}^{k}(\lambda) C_{i^{\prime} j^{\prime}}^{k^{\prime}}(\lambda)$. From this ambiguity it is clear that comparing the chiral ring i.e. the structure constants $C_{i j}^{k}$ or the 3 -point functions $C_{i j \bar{k}}$ at different points on $\mathcal{M}$ is not completely straightforward.

\footnotetext{
${ }^{6}$ More precisely we have $\mathcal{O}_{i} \propto \epsilon_{r i} \epsilon_{s j} \epsilon^{c d} \epsilon^{a b}\left\{Q_{c}^{r},\left[Q_{d}^{s},\left\{Q_{a}^{i},\left[Q_{b}^{j}, \Phi_{i}\right]\right\}\right]\right.$ where the constant of proportionality is a matter of conventions and can be adjusted to simplify certain equations.
} 
Is there a canonical way to choose a basis of operators over the moduli space and to eliminate this ambiguity? As we now explain the answer is negative. Operator mixing is an intrinsic property of the conformal field theory, completely determined by its dynamics, and it is impossible to gauge it away by an appropriate choice of basis.

One way to understand why the operator mixing is unavoidable is the following: under an infinitesimal deformation by a marginal operator $\mathcal{O}$ the correlators of the conformal field theory change by

$$
\int d^{4} x\left\langle\mathcal{O}(x) \varphi_{1}\left(z_{1}\right) \ldots \varphi_{n}\left(z_{n}\right)\right\rangle
$$

In general these integrated correlators are divergent due to short distance singularities in the OPE between $\mathcal{O}$ and the other insertions. This means that to define the deformed correlators we have to find a way to renormalize the divergent integral (3.4).

The renormalization method that we use is quite natural [23]: to first order in the perturbation we cut out small balls of radius $\varepsilon$ around the insertions $\varphi_{i}\left(x_{i}\right)$, and we compute the integrated correlator as a function of the cutoff $\varepsilon$. Then we expand the integrated correlator in powers of $\frac{1}{\varepsilon}$, throw away all the divergent pieces and keep the finite result as $\varepsilon \rightarrow 0$. This defines "renormalized" deformed correlators to first order in the deformation. Going to second order we basically do the same, but we have to be careful about the pieces that we already subtracted at first order. This approach was discussed in [24, 25] and nicely developed for deformations of two-dimensional conformal field theories in [23], to which we refer the reader for more details. Again a careful analysis shows that if we apply this procedure twice to compute the second order perturbation we find that in general the following expression does not vanish

$$
\int d^{4} x \int d^{4} y\left\langle\mathcal{O}_{[\mu}(x) \mathcal{O}_{\nu]}(y) \varphi_{1}\left(z_{1}\right) \ldots \varphi_{n}\left(z_{n}\right)\right\rangle \neq 0
$$

indicating that the two deformations do not commute, i.e. there is curvature on the space of operators. In other words, the operator mixing is inevitable because there is no way one can consistently renormalize the deformed correlators and at the same time avoid (3.5). ${ }^{7}$

\footnotetext{
${ }^{7}$ Sometimes this discussion is presented in terms of "contact terms" in the OPE between $\mathcal{O}$ and the other operators. The contact terms are tuned in such a way as to cancel the divergent parts of the integrated correlator, but may also give finite contributions which are interpreted as the connection on the space of operators $[26,27]$.

In a sense the contact terms are not an intrinsic notion in a fixed CFT, before we consider perturbing it. In a given CFT only correlators of operators at distinct points are meaningful. The contact terms have to be introduced when we want to consider integrated correlators which, as we discussed, require the use of some renormalization method. The contact terms are then chosen in such a way that they impose our preferred renormalization procedure in all correlators in a consistent way, and allow us to do the integrals such as (3.4) including the coincident points.

On the other hand if we decide to explicitly use our renormalization prescription [23] whenever we deal with deformed correlators, then we will never have to bring two operators at exactly the same point and we do not need to worry about contact terms. Said differently, the contact terms do not have to be included in the renormalization method, but rather they are determined by it. In the rest of the paper we will follow the prescription of [23] which was described above and will work in terms of regularized and renormalized integrated correlators, so we will not have to talk about contact terms anymore. We would like to thank K. Skenderis for discussions about the contact terms.
} 


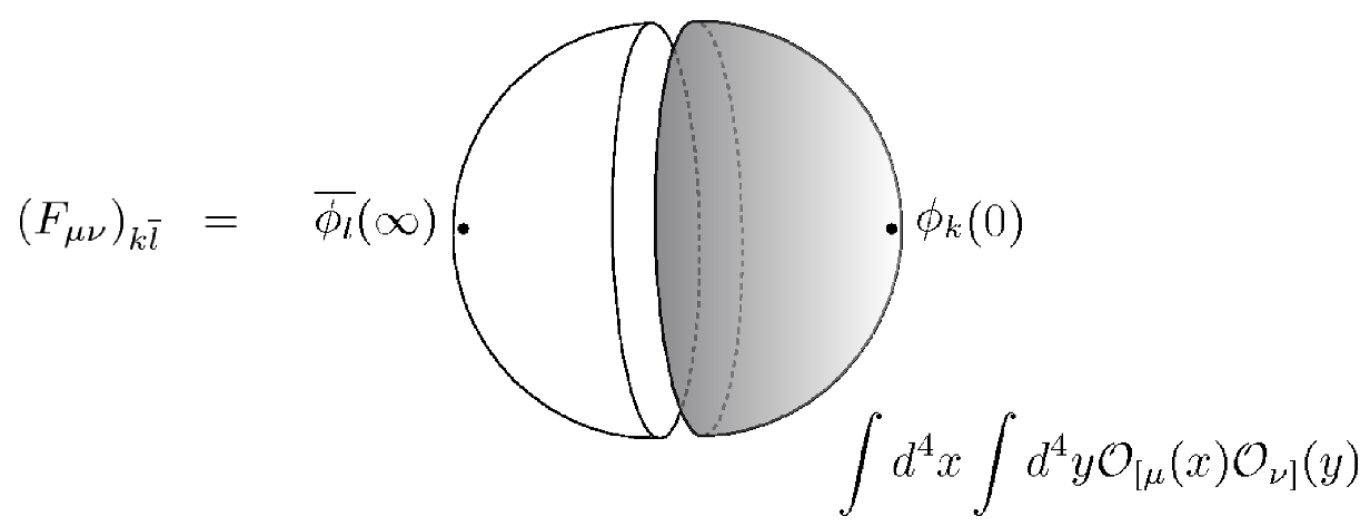

Figure 1. Computation of operator mixing from a 4-point function.

Because of the operator mixing we should be thinking of chiral primaries as operators taking values in vector bundles over the moduli space of the theory. These bundles have nontrivial curvature. Let us call $\mathcal{V}_{R}$ the bundle of the chiral primaries of $\mathrm{U}(1)_{\mathrm{R}}$ charge $R$. Then the chiral ring coefficients describe the OPE multiplication between different bundles

$$
C_{i j}^{k}: \mathcal{V}_{R} \otimes \mathcal{V}_{R^{\prime}} \rightarrow \mathcal{V}_{R+R^{\prime}}
$$

Each of the $\mathcal{V}_{R}$ bundles has nontrivial connection which encodes the operator mixing. For a set of chiral primaries of the same charge $\phi_{i}$ which can mix under motion on $\mathcal{M}$ we describe the mixing by the connection $A_{\mu i}^{j}$ as $\delta_{\mu} \phi_{i}=A_{\mu i}^{j} \phi_{j}$ and we have the covariant derivative

$$
\nabla_{\mu}=\partial_{\mu}-A_{\mu}
$$

and curvature

$$
F_{\mu \nu} \equiv\left[\nabla_{\mu}, \nabla_{\nu}\right]=\partial_{\mu} A_{\nu}-\partial_{\nu} A_{\mu}+\left[A_{\mu}, A_{\nu}\right]
$$

The connection is determined by the dynamics of the conformal field theory by the following condition

$$
\nabla_{\mu}\left\langle\varphi_{1}\left(z_{1}\right) \ldots \varphi_{n}\left(z_{n}\right)\right\rangle=\left[\int d^{4} x\left\langle\mathcal{O}_{\mu}(x) \varphi_{1}\left(z_{1}\right) \ldots \varphi_{n}\left(z_{n}\right)\right\rangle\right]_{\text {renormalized }}
$$

where $\varphi_{i}$ are general local operators (not necessarily chiral primary) and the renormalization prescription is the one we mentioned earlier. From now on, whenever we write integrated correlators we will always refer to their "renormalized" values, so we will drop the "renormalized" subscript from the integrals.

Starting from (3.6) it is straightforward to find an expression for the curvature of the bundle of chiral primary operators. We consider deformations by two marginal operators $\mathcal{O}_{\mu}, \mathcal{O}_{\nu}$ and compute the infinitesimal variation of an operator $\phi_{k}$ under the deformation by the antisymmetrized combination $\mathcal{O}_{[\mu}(x) \mathcal{O}_{\nu]}(y)$. Then we project this variation on the space of operators with which $\phi_{k}$ mixes. In terms of correlators this can be computed by taking the 4-sphere $\mathbf{S}^{4}$, dividing it into two hemispheres, inserting $\phi_{k}$ at the center of one hemisphere and the conjugate operator $\bar{\phi}_{l}$ on the other hemisphere and then integrating the 
combination $\int d^{4} x \int d^{4} y \mathcal{O}_{[\mu}(x) \mathcal{O}_{\nu]}(y)$ on the hemisphere where $\phi_{k}$ was inserted, as depicted in figure 1. Mapping the $\mathbf{S}^{4}$ onto $\mathbf{R}^{4}$ we find that the curvature can also be written as

$$
\left(F_{\mu \nu}\right)_{k \bar{l}}=\int_{|x| \leq 1} d^{4} x \int_{|y| \leq 1} d^{4} y\left\langle\bar{\phi}_{l}(\infty) \mathcal{O}_{[\mu}(x) \mathcal{O}_{\nu]}(y) \phi_{k}(0)\right\rangle
$$

where again the double integral is supposed to be renormalized by the prescription that we mentioned earlier, see also $[18,23]$ for more details. From the curvature it is possible to reconstruct the connection $A_{\mu i}^{j}$, at least locally. Once this connection has been computed, then it is meaningful to ask what is the dependence of the chiral ring coefficients on the coupling constants.

To give a simple example why all of the previous analysis was necessary, let us consider a theory where we believe that the 3-point functions of chiral primaries are "independent of the coupling constant". According to what we discussed this statement should be interpreted as $\nabla_{\mu} C_{i j}^{k}=0$ and not $\partial_{\mu} C_{i j}^{k}=0$ which is a non-covariant, basis-dependent equation. What this means in practice is that in a theory where $F_{\mu \nu} \neq 0$, it is impossible to choose a basis such that $\partial_{\mu} C_{i j}^{k}=0$ everywhere on the moduli space, while it is still possible to have $\nabla_{\mu} C_{i j}^{k}=0$. This is the right way to express the notion that the 3 -point functions are "independent of the coupling constant".

Let us make a few more comments. In all of the above we have assumed that the conformal dimensions of the operators do not change as we vary the coupling constants. This assumption was motivated because we are interested in correlation functions of chiral primary operators, whose conformal dimension cannot change as long as they remain "chiral primaries". 8 If we consider more general operators whose conformal dimensions vary continuously, then the deformed correlators can have logarithmic terms and we have to be more careful in defining the connection.

Finally, the connection defined above is compatible with the Zamolodchikov metric defined by the 2-point functions of chiral primaries

$$
\nabla_{\mu} g_{k \bar{l}} \equiv \nabla_{\mu}\left\langle\bar{\phi}_{l}(\infty) \phi_{k}(0)\right\rangle=\int d^{4} x\left\langle\mathcal{O}_{\mu}(x) \bar{\phi}_{l}(\infty) \phi_{k}(0)\right\rangle=0
$$

since the 3-point function in the integrand vanishes.

\section{Marginal deformations in $\mathcal{N}=2$ superconformal field theories}

We now focus on the main goal of this paper, the analysis of marginal deformations of $\mathcal{N}=2$ superconformal field theories in four dimensions. The important element is that in

\footnotetext{
${ }^{8}$ Consider an operator $\phi$ which is chiral primary everywhere on the moduli space, so it satisfies $\Delta=\frac{R}{2}$ everywhere. Can its R-charge be a function of the coupling constants? Since it is an abelian charge one may wonder whether it can change continuously and simultaneously with $\Delta$, preserving the condition $\Delta=\frac{R}{2}$. However it is easy to use a superconformal Ward identity to show that the 3-point function $\langle\mathcal{O} \phi \bar{\phi}\rangle$ vanishes, which means that the conformal dimension (and hence R-charge) of a chiral primary cannot change continuously on the moduli space. Notice that the discussion in this paragraph is not about whether chiral primaries can combine into long multiplets and lift from the unitarity bound $\Delta=\frac{R}{2}$, which is not excluded by this argument. We have ignored this effect because it can only happen at special points of the moduli space. We would like to thank J. de Boer for discussions on these issues.
} 
these theories the marginal operators are descendants of chiral primaries (3.1). As a result, the variation of the chiral ring under marginal deformations is tightly constrained. In this section we present the main results of our paper and leave some of the derivations for the next section and the appendices.

\subsection{Holomorphy of the chiral ring and WDVV equations}

We start by analyzing the moduli dependence of 3-point functions of chiral primaries. We consider a deformation of the theory ${ }^{9}$

$$
S \rightarrow S+\delta \lambda^{m} \int d^{4} x \mathcal{O}_{m}(x)+\delta \bar{\lambda}^{m} \int d^{4} x \overline{\mathcal{O}}_{m}(x)
$$

We denote by $\nabla_{m}, \bar{\nabla}_{m}$ the derivatives with respect to holomorphic and antiholomorphic marginal operators

$$
\left(\nabla_{m}\right)_{k}^{l}=\delta_{k}^{l} \partial_{m}-A_{m k}^{l}
$$

where $A_{m k}^{l}$ is the connection.

First we notice that the chiral ring coefficients are (covariantly) holomorphic

$$
\bar{\nabla}_{m} C_{i j}^{k}=0
$$

which is a consequence ${ }^{10}$ of the vanishing of the 4-point function

$$
\left\langle\overline{\mathcal{O}}_{m}(x) \bar{\phi}_{l}(\infty) \phi_{i}(1) \phi_{j}(0)\right\rangle=0
$$

This a direct result of the superconformal Ward identity (2.3): we notice that the marginal operator is of the form $\overline{\mathcal{O}}_{m}=\bar{Q}^{4} \cdot \bar{\Phi}_{m}$. Since all supercharges $\bar{Q}_{i \dot{a}}$ anticommute among themselves, we can pick one of them, call it $\overline{\mathbf{Q}}$, and pull it to the left of the other supercharges. So we write the marginal operator as

$$
\overline{\mathcal{O}}_{m}(x)=\{\overline{\mathbf{Q}}, \Lambda\}(x)
$$

for some operator $\Lambda$. Now according to $(2.3)$ we move $\overline{\mathbf{Q}}$ to the other insertions. We do not get any contribution from $\phi_{i}(1)$ and $\phi_{j}(0)$ since they are chiral primaries and are both annihilated by $\overline{\mathbf{Q}}$. We do not get any contribution from $\bar{\phi}_{l}(\infty)$ because as we explained there is no contribution from the point at infinity if the operator is a superconformal primary. So we find the desired relation (4.2). Notice that in this proof we have assumed that all points are distinct $x \neq\{0,1, \infty\}$ and according to the discussion in section 3 we do not have to worry about contact terms.

Notice that this argument did not depend on the number of chiral primaries inserted in the correlator, since we only needed to "hide" the antichiral operator $\bar{\phi}_{l}$ at infinity. So it can be used to show that more general "extremal correlators" are also covariantly holomorphic

$$
\bar{\nabla}_{m}\left\langle\phi_{1}\left(x_{1}\right) \ldots \phi_{n}\left(x_{n}\right) \bar{\phi}_{n+1}(z)\right\rangle=0
$$

\footnotetext{
${ }^{9}$ This is only schematic, we do not assume that there is an "action" or that the CFT has a Lagrangian description. By this notation we just mean that we consider a deformation by a real linear combination of the marginal operators $\mathcal{O}_{m}$ and $\overline{\mathcal{O}}_{m}$.

${ }^{10}$ From the vanishing of the 4-point function follows that $\bar{\nabla}_{m} C_{i j \bar{l}}=0$. Then using that $\bar{\nabla}_{m} g^{\bar{l} k}=0$ from (3.8) and $C_{i j}^{k}=C_{i j \bar{l}} g^{\bar{l} k}$ we get (4.1).
} 
where the $\mathrm{U}(1)_{\mathrm{R}}$ charges satisfy $R_{n+1}=\sum_{i=1}^{n} R_{i}$. If we have two or more antichiral insertions then we cannot apply a similar argument.

Now let us consider the holomorphic dependence of 3-point functions of chiral primaries and show how to derive the four-dimensional analogue of the WDVV equation [14-16]. In general we have $\left\langle\mathcal{O}_{m}(x) \bar{\phi}_{k}(\infty) \phi_{i}(1) \phi_{j}(0)\right\rangle \neq 0$ so the $C_{i j}^{k}$ can have non-trivial holomorphic dependence on the coordinates $\lambda$ of $\mathcal{M}$

$$
\nabla_{m} C_{i j}^{k} \neq 0
$$

We can however prove the integrability condition

$$
\nabla_{i} C_{j k}^{l}=\nabla_{j} C_{i k}^{l}
$$

where we assumed that the indices $i, j$ correspond to chiral primaries of $\Delta=2$. This is a result of the following relation of 4-point functions

$$
\left\langle\mathcal{O}_{i}(x) \bar{\phi}_{l}(\infty) \phi_{j}(1) \phi_{k}(0)\right\rangle=\left\langle\mathcal{O}_{j}(x) \bar{\phi}_{l}(\infty) \phi_{i}(1) \phi_{k}(0)\right\rangle
$$

This can be derived in similar manner using the superconformal Ward identities by moving the supercharges from $\mathcal{O}_{i}$ onto $\phi_{j}$, which they transform into $\mathcal{O}_{j}$ (the contribution from $\phi_{k}$ can be killed according to the Ward identity in appendix A). Then by acting with a conformal transformation we can switch the positions of the operators $\phi_{i}$ and $\mathcal{O}_{j}$ leaving the other two unchanged.

Next we consider the associativity of the chiral ring. We start with the following 4-point function of chiral primaries

$$
G(x, y)=\left\langle\bar{\phi}_{l}(\infty) \phi_{i}(x) \phi_{j}(y) \phi_{k}(0)\right\rangle
$$

where we take $\phi_{i}, \phi_{j}$ to be Lorentz scalars. Since the OPE of chiral primaries is nonsingular we have

$$
\lim _{x \rightarrow 0} \lim _{y \rightarrow 0} G(x, y)=C_{j k}^{m} C_{i m \bar{l}}
$$

and

$$
\lim _{y \rightarrow 0} \lim _{x \rightarrow 0} G(x, y)=C_{i k}^{m} C_{j m \bar{l}}
$$

Associativity of the OPE (or crossing symmetry) implies that the two limits should be the same. Multiplying with the inverse metric we get the desired expression

$$
C_{i k}^{m} C_{j m}^{l}=C_{j k}^{m} C_{i m}^{l}
$$

Before we proceed, let us summarize the results we have found so far

$$
\begin{aligned}
\bar{\nabla}_{j} C_{k} & =0, & \nabla_{i} \bar{C}_{k} & =0 \\
\nabla_{i} C_{j} & =\nabla_{j} C_{i}, & \bar{\nabla}_{i} \bar{C}_{j} & =\bar{\nabla}_{j} \bar{C}_{i} \\
{\left[C_{i}, C_{j}\right] } & =0, & \left.\bar{C}_{i}, \bar{C}_{j}\right] & =0
\end{aligned}
$$


where the notation $C_{i}$ means that we think of it as a matrix $\left(C_{i}\right)_{k}^{l}$. These results are identical to those found in two-dimensional superconformal field theories. ${ }^{11}$

The WDVV equations in $\mathcal{N}=2$ theories in four dimensions have also been discussed in [28-31]. These papers focused on the prepotential of the low energy effective action on the Coulomb branch of Seiberg-Witten theories, while we study the dependence of correlators on the position on the moduli space of the CFT. It would be nice to clarify the relation to our results.

\subsection{The $t t^{*}$ equations}

So far we have seen that the 3-point functions are, roughly speaking, holomorphic functions of the coupling constants. Remarkably more constraints can be found by computing the connection $A_{m k}^{l}$ on the bundle of chiral primaries. From the point of view of conformal perturbation theory this connection describes the operator mixing under marginal deformations. Its curvature is computed by (3.7), where the two marginal operators are now descendants of chiral primaries.

As we show in the next section, in the case of $\mathcal{N}=2$ superconformal field theories, we can actually perform the double integral of (3.7) and we find the following simple expressions for the curvature ${ }^{12}$

$$
\begin{aligned}
{\left[\nabla_{i}, \nabla_{j}\right]_{k}^{l} } & =0 \\
{\left[\bar{\nabla}_{i}, \bar{\nabla}_{j}\right]_{k}^{l} } & =0 \\
\frac{1}{(2 \pi)^{4}}\left[\nabla_{i}, \bar{\nabla}_{j}\right]_{k}^{l} & =-\left[C_{i}, \bar{C}_{j}\right]_{k}^{l}+g_{i \bar{j}} \delta_{k}^{l}\left(1-\frac{3 R}{4 c}\right)
\end{aligned}
$$

where in these equations the curvature operators are acting on the subspace of chiral primaries of $\mathrm{U}(1)_{\mathrm{R}}$ charge $R$ which are labeled by the indices $k, l$, while the indices $i, j$ denote the marginal operators. The constant $c$ is the central charge of the conformal field theory, defined by the 2-point function of the stress-energy tensor, $g_{i \bar{j}}$ is proportional to the metric on the moduli space (see $(3.3))$ and we use a condensed notation for $\left[C_{i}, \bar{C}_{j}\right]_{k}^{l}=$ $C_{i k}^{r} g_{r \bar{m}} C_{\bar{j} \bar{p}}^{* \bar{m}} g^{\bar{p} l}-g_{k \bar{r}} C_{\bar{j} \bar{p}}^{* \bar{p}} g^{\bar{p} n} C_{\mathrm{in}}^{l}$.

These equations are the main results of our analysis. They were first discovered in the context of two-dimensional $\mathcal{N}=(2,2)$ supersymmetric field theories and called " $t t^{*}$ equations" [17]. The first two equations show that the bundles of chiral primaries $\mathcal{V}_{p}$ are

\footnotetext{
${ }^{11}$ As in two-dimensional topological field theories, we could consider the topologically twisted $\mathcal{N}=2$ theory and define the "topological metric" by the 2-point function $\eta_{i j} \equiv\left\langle\phi_{i} \phi_{j}\right\rangle_{\text {twisted }}$ on $\mathbf{S}^{4}$ and the 3-point functions with all indices lowered $C_{i j k} \equiv\left\langle\phi_{i} \phi_{j} \phi_{k}\right\rangle_{\text {twisted }}$. Then the integrability equation (4.4) implies that a scalar function $\mathcal{F}$ exists (locally), such that $\eta_{i j}=\partial_{i} \partial_{j} \mathcal{F}(\lambda), C_{i j k}=\partial_{i} \partial_{j} \partial_{k} \mathcal{F}(\lambda)$ and the associativity condition (4.5) can be written as a nonlinear differential equation

$$
\frac{\partial^{3} \mathcal{F}}{\partial \lambda_{i} \partial \lambda_{k} \partial \lambda_{m}}\left(\frac{\partial^{2} \mathcal{F}}{\partial \lambda_{m} \partial \lambda_{n}}\right)^{-1} \frac{\partial^{3} \mathcal{F}}{\partial \lambda_{n} \partial \lambda_{j} \partial \lambda_{l}}=\frac{\partial^{3} \mathcal{F}}{\partial \lambda_{j} \partial \lambda_{k} \partial \lambda_{m}}\left(\frac{\partial^{2} \mathcal{F}}{\partial \lambda_{m} \partial \lambda_{n}}\right)^{-1} \frac{\partial^{3} \mathcal{F}}{\partial \lambda_{n} \partial \lambda_{i} \partial \lambda_{l}}
$$

This and other properties of the topologically twisted $\mathcal{N}=2$ SCFTs in four dimensions will be discussed in $[19]$.

${ }^{12}$ We tried to keep all conventions consistent and to determine the numerical factors exactly, but we have not found an independent way to verify the factor $3 / 4$.
} 
holomorphic vector bundles, while the last one allows us to compute the curvature in terms of the chiral ring coefficients $C_{i j}^{k}$.

The set of equations (4.6) and (4.7) can be made less abstract by working in a "holomorphic gauge": from the second equation in (4.7) we see that it is possible to choose the basis of chiral primaries to depend on the coupling constants in such a way that $A_{\bar{j} k}^{l}=0$. In that basis the covariant and ordinary antiholomorphic derivatives coincide $\nabla_{\bar{j}}=\partial_{\bar{j}}$ and equation (4.1) becomes

$$
\partial_{\bar{m}} C_{i j}^{k}=0
$$

So in this basis the chiral ring coefficients become ordinary holomorphic functions of the moduli (and not just "covariantly holomorphic"). If we know the global structure of the moduli space $\mathcal{M}$ and the behavior of the chiral ring coefficients in the weak-coupling limits it may be possible to completely determine them for all values of the coupling.

A further simplification in the holomorphic gauge is that the connection and curvature can be directly written in terms of the Zamolodchikov metric for chiral primaries

$$
\begin{aligned}
A_{i k}^{l} & =g^{\overline{m l}} \partial_{i} g_{k \bar{m}} \\
\left(F_{i \bar{j}}\right)_{k}^{l} & =\partial_{\bar{j}} A_{i k}^{l}=\partial_{\bar{j}}\left(g^{\bar{m} l} \partial_{i} g_{k \bar{m}}\right)
\end{aligned}
$$

where we used the fact that the bundles are holomorphic and that the metric and connection are hermitian and compatible. ${ }^{13}$ Then we find that the integrability equations (4.6) become

$$
\partial_{i} C_{j k}^{l}-\partial_{j} C_{i k}^{l}=\left[g^{-1} \partial_{i} g, C_{j}\right]_{k}^{l}-\left[g^{-1} \partial_{j} g, C_{i}\right]_{k}^{l}
$$

and the last of the $t t^{*}$ equations (4.7) can be written as

$$
\frac{1}{(2 \pi)^{4}} \bar{\partial}_{j}\left(g_{k \bar{m}} \partial_{i} g^{\bar{m} l}\right)=-\left[C_{i}, \bar{C}_{j}\right]_{k}^{l}+g_{i \bar{j}} \delta_{k}^{l}\left(1-\frac{3 R}{4 c}\right)
$$

When written in the form (4.8), (4.10) and (4.11), the $t t^{*}$ equations can be considered as partial differential equations relating the 2- and 3-point functions $g_{k \bar{l}}(\lambda)$ and $C_{i j}^{k}(\lambda)$ of chiral primaries over the moduli space. One of their applications is that they allow us to determine the Zamolodchikov metric $g_{k \bar{l}}(\lambda)$ (for the entire tower of chiral primaries) for all values of the coupling. The important point is that while the 2-point functions $g_{k \bar{l}}$ are not holomorphic, they can be computed as solutions of these classical differential equations whose coefficients are the holomorphic functions $C_{i j}^{k}(\lambda)$, which are in general easier to determine.

\subsection{A flat connection}

Let us briefly comment on the perhaps unfamiliar term $g_{i \bar{j}} g_{k \bar{l}}\left(1-\frac{3 R}{4 c}\right)$ in the third equation of (4.7), which was absent in the original derivation [17]. There the connection for the Ramond ground states of the topologically twisted theory was computed. In our case we are computing the connection characterizing operator mixing in the conformal field

\footnotetext{
${ }^{13}$ From the compatibility of the metric with the connection we have $\nabla_{i} g_{k}=0$. Using that in the holomorphic gauge we have $A_{\bar{j} k}^{l}=A_{j \bar{k}}^{\bar{l}}=0$ we can also write the compatibility condition as $\partial_{i} g_{k \bar{m}}-A_{i k}^{l} g_{l \bar{m}}=$ 0 and then (4.9) follows.
} 
theory. Apparently there are some different phase factors in the definitions of these two connections. To check that in our case we do need this extra term we notice that without it we would have nontrivial holonomy (phase) for the identity operator in the CFT (see also [18]), which seems unnatural.

In any case it is not difficult to relate the two results. Let us call $\mathcal{K}$ the holomorphic line bundle whose curvature is proportional to the Kähler form on the moduli space

$$
F_{i j}=F_{\overline{i j}}=0, \frac{1}{(2 \pi)^{4}} F_{i \bar{j}}=g_{i \bar{j}}
$$

And let us redefine each of the bundles of chiral primaries $\mathcal{V}$ in the following way $V^{\prime}=$ $\mathcal{K}^{\left(\frac{3 R}{4 c}-1\right)} \otimes V$. Then the curvature for the bundles $V^{\prime}$ will be

$$
\begin{aligned}
& {\left[\nabla_{i}^{\prime}, \nabla_{j}^{\prime}\right]_{k \bar{l}} }=0 \\
& {\left[\bar{\nabla}_{i}^{\prime}, \bar{\nabla}_{j}^{\prime}\right]_{k \bar{l}} }=0 \\
& \frac{1}{(2 \pi)^{4}}\left[\nabla_{i}^{\prime}, \bar{\nabla}_{j}^{\prime}\right]_{k \bar{l}}=-\left[C_{i}, \bar{C}_{j}\right]_{k \bar{l}}
\end{aligned}
$$

which is the same as the one discussed in [17].

Finally let us mention that the $t t^{*}$ equations, together with (4.6), are equivalent to the statement that one can define an improved connection

$$
D_{i}=\nabla_{i}^{\prime}-C_{i}
$$

The improved connection is flat

$$
\left[D_{i}, D_{j}\right]=\left[\bar{D}_{i}, \bar{D}_{j}\right]=\left[D_{i}, \bar{D}_{j}\right]=0
$$

However this connection is not compatible with the Zamolodchikov metric.

\section{Derivation of the four-dimensional $t t^{*}$ equations}

Now we show how the four-dimensional $t t^{*}$ equations can be derived from the point of view of conformal perturbation theory. Let us start with the first equation $\left[\nabla_{i}, \nabla_{j}\right]_{k}^{l}=0$. For this it is sufficient to show that

$$
\left\langle\bar{\phi}_{l}(\infty) \mathcal{O}_{i}(x) \mathcal{O}_{j}(y) \phi_{k}(0)\right\rangle=0
$$

We first act with a conformal transformation to interchange the points at $\infty$ and 0 , so equivalently we want to prove the vanishing of $\left\langle\phi_{k}(\infty) \mathcal{O}_{i}(x) \mathcal{O}_{j}(y) \bar{\phi}_{l}(0)\right\rangle$. This is easy to prove as before. We write $\mathcal{O}_{i}=\left\{\mathbf{Q}, \Lambda^{\prime}\right\}$ and then using (2.3) we move $\mathbf{Q}$ to the other insertions. We find that none of the terms contribute (where we also have to use that $\left[\mathbf{Q}, \mathcal{O}_{j}\right]=0$ ). So we find that the 4 -point function vanishes. Similarly we show that $\left[\bar{\nabla}_{i}, \bar{\nabla}_{j}\right]_{k}^{l}=0$.

We will now show how to derive the third of the $t t^{*}$ equations (4.7). For that we need to compute the following integrated 4-point function

$$
\left(F_{i \bar{j}}\right)_{k \bar{l}} \equiv\left[\nabla_{i}, \bar{\nabla}_{j}\right]_{k \bar{l}}=\int_{|x| \leq 1} d^{4} x \int_{|y| \leq 1} d^{4} y\left\langle\bar{\phi}_{l}(\infty) \mathcal{O}_{[i}(x) \overline{\mathcal{O}}_{j]}(y) \phi_{k}(0)\right\rangle
$$


The superconformal Ward identities of the $\mathcal{N}=2$ CFT allow us to write

$$
\left\langle\bar{\phi}_{l}(\infty) \mathcal{O}_{i}(x) \overline{\mathcal{O}}_{j}(y) \phi_{k}(0)\right\rangle=\nabla_{x}^{2} \nabla_{x}^{2}\left\langle\bar{\phi}_{l}(\infty) \Phi_{i}(x) \bar{\Phi}_{j}(y) \phi_{k}(0)\right\rangle
$$

where $\nabla_{x}^{2}$ is the four-dimensional Laplacian with respect to $x$. A quick explanation of this identity is as follows: we have $\overline{\mathcal{O}}_{j}=\bar{Q}^{4} \cdot \bar{\Phi}_{j}$. We want to move the $\bar{Q}$ 's away from the operator $\bar{\Phi}_{j}(y)$ using $(2.3)$. The operator $\phi_{k}$ is annihilated by the $\bar{Q}$ 's being an antichiral primary and $\bar{\phi}_{l}$ does not contribute according to (2.3), so the only contribution will be when the $\bar{Q}$ 's hit the $\mathcal{O}_{i}$. Then we end up with something of the form $\bar{Q}^{4} \cdot Q^{4} \cdot \Phi_{i}(x)$. From the supersymmetry algebra $\left\{Q_{a}^{i}, \bar{Q}_{j, \dot{b}}\right\}=2 \delta_{j}^{i} P_{a \dot{b}}$ we see that we get derivatives $\partial_{x}$ when we anticommute the supercharges. The only Lorentz invariant combination we can construct out of four $\partial_{x}$ 's is $\nabla_{x}^{2} \nabla_{x}^{2}$. So we have

$$
\begin{aligned}
\left(F_{i \bar{j}}\right)_{k \bar{l}}= & \int_{|x| \leq 1} d^{4} x \int_{|y| \leq 1} d^{4} y \\
& \times\left(\nabla_{x}^{2} \nabla_{x}^{2}\left\langle\bar{\phi}_{l}(\infty) \Phi_{i}(x) \bar{\Phi}_{j}(y) \phi_{k}(0)\right\rangle-\nabla_{y}^{2} \nabla_{y}^{2}\left\langle\bar{\phi}_{l}(\infty) \Phi_{i}(y) \bar{\Phi}_{j}(x) \phi_{k}(0)\right\rangle\right)
\end{aligned}
$$

Now we use the following conformal Ward identity ${ }^{14}$

$$
\nabla_{x}^{2} \nabla_{x}^{2}\left\langle\bar{\phi}_{l}(\infty) \Phi_{i}(x) \bar{\Phi}_{j}(y) \phi_{k}(0)\right\rangle=\nabla_{y}^{2} \nabla_{y}^{2}\left(\frac{|y|^{4}}{|x|^{4}}\left\langle\bar{\phi}_{l}(\infty) \Phi_{i}(x) \bar{\Phi}_{j}(y) \phi_{k}(0)\right\rangle\right)
$$

Using this result and the OPEs between chiral and antichiral primaries (appendix D) we can see that the integrand in (5.1) is smooth as $y \rightarrow 0$, so doing the $y$-integration

$$
\begin{aligned}
\left(F_{i \bar{j}}\right)_{k \bar{l}}= & \int_{|x| \leq 1} d^{4} x \int_{|y|=1} d \Omega_{3}^{y}|y|^{2}\left(y \cdot \partial_{y}\right) \\
& \times\left(\nabla_{y}^{2} \frac{|y|^{4}}{|x|^{4}}\left\langle\bar{\phi}_{l}(\infty) \Phi_{i}(x) \bar{\Phi}_{j}(y) \phi_{k}(0)\right\rangle-\nabla_{y}^{2}\left\langle\bar{\phi}_{l}(\infty) \Phi_{i}(y) \bar{\Phi}_{j}(x) \phi_{k}(0)\right\rangle\right)
\end{aligned}
$$

Now we use two more conformal Ward identities ${ }^{15}$

$$
\nabla_{y}^{2}\left\langle\bar{\phi}_{l}(\infty) \Phi_{i}(y) \bar{\Phi}_{j}(x) \phi_{k}(0)\right\rangle=\nabla_{x}^{2}\left(\frac{|x|^{2}}{|y|^{2}}\left\langle\bar{\phi}_{l}(\infty) \Phi_{i}(y) \bar{\Phi}_{j}(x) \phi_{k}(0)\right\rangle\right)
$$

and

$$
\begin{aligned}
& \int_{|x|=\mathrm{const}} d \Omega_{3}^{x} \nabla_{y}^{2}\left(|y|^{4}\left\langle\bar{\phi}_{l}(\infty) \Phi_{i}(x) \bar{\Phi}_{j}(y) \phi_{k}(0)\right\rangle\right)= \\
& \int_{|x|=\mathrm{const}} d \Omega_{3}^{x}|y|^{2}\left(x \cdot \partial_{x}\right)\left(|x|^{2}\left(x \cdot \partial_{x}\right)\left(\frac{1}{|x|^{2}}\left\langle\bar{\phi}_{l}(\infty) \Phi_{i}(x) \bar{\Phi}_{j}(y) \phi_{k}(0)\right\rangle\right)\right)
\end{aligned}
$$

\footnotetext{
${ }^{14}$ This identity is true for scalar conformal primaries $\Phi_{i}, \bar{\Phi}_{j}$ of dimension $\Delta=2$ and for scalar conformal primaries $\phi_{k}, \bar{\phi}_{l}$ of any (but same) conformal dimension. If $\phi_{k}, \bar{\phi}_{l}$ are primaries with nonzero spin, then the identity is still true after integrating over the angular directions of the variable $x$. It does not depend on supersymmetry. The easiest way to check it is to consider the double OPE of both sides taking say $x$ close to $\infty$ and $y$ close to 0 .

${ }^{15}$ Same is true as in the previous footnote.
} 


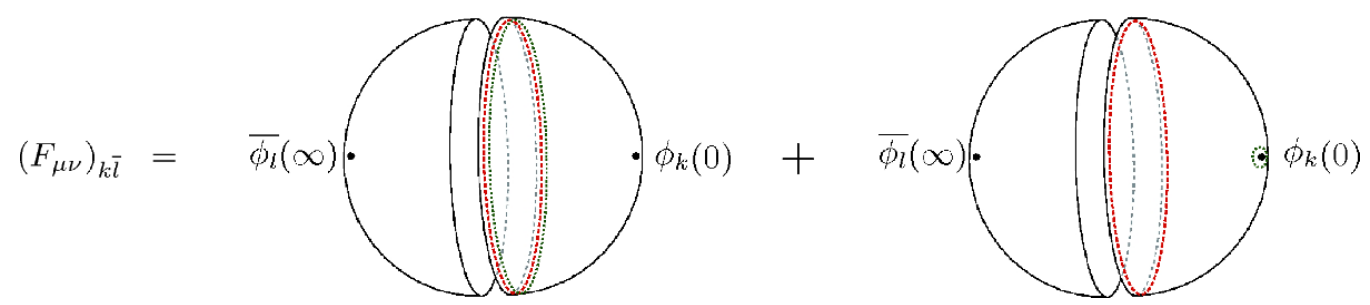

Figure 2. The colored dotted lines denote surface integrals of chiral primary operators and their conjugates.

Substituting (5.3) and (5.4) into (5.2) and doing the $x$ integral we find

$$
\begin{aligned}
\left(F_{i \bar{j}}\right)_{k \bar{l}}= & \lim _{r \rightarrow 1^{-}} \int_{|x|=r} d \Omega_{3}^{x} \int_{|y|=1} d \Omega_{3}^{y}|x|^{2}|y|^{2}\left(y \cdot \partial_{y}\right)\left(x \cdot \partial_{x}\right) \\
& \left(\frac{|y|^{2}}{|x|^{2}}\left\langle\bar{\phi}_{l}(\infty) \Phi_{i}(x) \bar{\Phi}_{j}(y) \phi_{k}(0)\right\rangle-\frac{|x|^{2}}{|y|^{2}}\left\langle\bar{\phi}_{l}(\infty) \Phi_{i}(y) \bar{\Phi}_{j}(x) \phi_{k}(0)\right\rangle\right) \\
- & \lim _{r \rightarrow 0} \int_{|x|=r} d \Omega_{3}^{x} \int_{|y|=1} d \Omega_{3}^{y}|x|^{2}|y|^{2}\left(y \cdot \partial_{y}\right)\left(x \cdot \partial_{x}\right) \\
& \left(\frac{|y|^{2}}{|x|^{2}}\left\langle\bar{\phi}_{l}(\infty) \Phi_{i}(x) \bar{\Phi}_{j}(y) \phi_{k}(0)\right\rangle-\frac{|x|^{2}}{|y|^{2}}\left\langle\bar{\phi}_{l}(\infty) \Phi_{i}(y) \bar{\Phi}_{j}(x) \phi_{k}(0)\right\rangle\right)
\end{aligned}
$$

which is schematically depicted in figure 2 . The contribution from the limit $r \rightarrow 0$ can be easily evaluated by considering the OPE between the operator at $x$ and the one at 0 . We have

$$
\begin{aligned}
\Phi_{i}(x) \phi_{k}(0) & =C_{i k}^{m} \phi_{m}(0)+\ldots \\
\bar{\Phi}_{j}(x) \phi_{k}(0) & =g_{k \bar{r}} C_{\bar{j} \bar{p}}^{* \bar{p}} g^{\bar{p} n} \frac{\phi_{n}(0)}{|x|^{4}}+\ldots
\end{aligned}
$$

where we did not include any higher order terms since they do not contribute to the integral in the limit $r \rightarrow 0$. Notice that the first expression is the chiral ring OPE while the second is explained in appendix D. So the contribution from this limit is

$$
(2 \pi)^{4} g_{k \bar{r}} C_{\bar{j} \bar{p}}^{* \bar{r}} g^{\bar{p} n} C_{\mathrm{in}}^{m} g_{m \bar{l}}-(2 \pi)^{4} C_{i k}^{r} g_{r \bar{m}} C_{\bar{j} \bar{l}}^{* \bar{m}}=-(2 \pi)^{4}\left[C_{i}, \bar{C}_{j}\right]_{k \bar{l}}
$$

Then for the contribution to (5.5) from the $r \rightarrow 1$ limit, we notice that there are many cancellations between the integrands at points related by $x \leftrightarrow y$ as $r \rightarrow 1$, and the only possible nonzero contribution is from the vicinity of the region $x=y$. There we are allowed to use the OPE between $\Phi_{i}$ and $\bar{\Phi}_{j}$. As we explain in appendix C, the only terms which do contribute are the identity operator and operators in the supermultiplet containing the stress-energy tensor. The contribution to the OPE of these operators is fixed by Ward identities so finally we find the following result

$$
(2 \pi)^{4} g_{i \bar{j}} g_{k \bar{l}}\left(1-\frac{3 R}{4 c}\right)
$$

Putting everything together we have

$$
\frac{1}{(2 \pi)^{4}}\left(F_{i \bar{j}}\right)_{k \bar{l}}=-\left[C_{i}, \bar{C}_{j}\right]+g_{i \bar{j}} g_{k \bar{l}}\left(1-\frac{3 R}{4 c}\right)
$$




\section{The Zamolodchikov metric on the moduli space}

The previous results allow us to find constraints for the geometry of the moduli space of general $\mathcal{N}=2$ superconformal field theories. In this section we show that the Zamolodchikov metric satisfies constraints similar to those of "special geometry". The marginal operators are of the form

$$
\mathcal{O}_{i}=Q^{4} \cdot \Phi_{i} \quad, \quad \overline{\mathcal{O}}_{j}=\bar{Q}^{4} \cdot \bar{\Phi}_{j}
$$

From this we see that the holonomy of the marginal operators is related to that of the chiral primaries of $\Delta=2$. But before we go on we have to explain a subtlety. The $\mathcal{N}=2$ superconformal algebra is invariant under a $\mathrm{U}(1)$ automorphism which rotates the left chiral supercharges as $Q \rightarrow e^{i \theta} Q$ and the right chiral ones as $\bar{Q} \rightarrow e^{-i \theta} \bar{Q}$ leaving all bosonic generators unchanged. ${ }^{16}$ This means that the phase of the supercharges is ambiguous and as we will see there is nontrivial holonomy for it under motion on the moduli space. In other words the left chiral supercharges are sections of a line bundle $\mathcal{L}$ and the right chiral supercharges $\bar{Q}$ of its conjugate $\overline{\mathcal{L}}$.

The curvature of $\mathcal{L}$ is the same as that of the left chiral supercurrents $G_{a \mu}^{i}$, since $Q_{a}^{i}=$ $\int d^{3} x G_{a 0}^{i}$. So we can use the general formula (3.7) for the curvature of the supercurrents to compute the curvature of $\mathcal{L}$

$$
F_{\mu \nu}^{\mathcal{L}}=\int_{|x| \leq 1} d^{4} x \int_{|y| \leq 1} d^{4} y\left\langle\bar{G}(\infty) \mathcal{O}_{[\mu}(x) \mathcal{O}_{\nu]}(y) G(0)\right\rangle
$$

where for simplicity we did not write the spinor and flavor indices. The curvature can be computed by similar methods as those used in section 5 and after some work we find the following

$$
\begin{aligned}
F_{i j}^{\mathcal{L}} & =0 \\
F_{i j}^{\mathcal{L}} & =0 \\
\frac{1}{(2 \pi)^{4}} F_{i \bar{j}}^{\mathcal{L}} & =\frac{1}{12 c} g_{i \bar{j}}
\end{aligned}
$$

So we learn that $\mathcal{L}$ is a holomorphic vector bundle whose curvature is proportional to the Kähler form of the moduli space.

Now we are ready to proceed. The marginal operators $\mathcal{O}_{i}$ are sections of the holomorphic tangent bundle $\mathcal{T} \mathcal{M}$. From expression (6.1) we see that we have

$$
\mathcal{T} \mathcal{M}=\mathcal{L}^{4} \otimes \mathcal{V}
$$

where $\mathcal{L}$ is the $\mathrm{U}(1)$ bundle of the supercharges and $\mathcal{V}$ is the vector bundle of the chiral primaries $\Phi_{i}$ of $\Delta=2, R=4$. The Riemann tensor on the moduli space is determined by the curvature of $\mathcal{T} \mathcal{M}$, so it will be the sum of the curvatures of the bundles $\mathcal{V}$ and that of $\mathcal{L}^{4}$. The curvature of $\mathcal{V}$ is given by the $t t^{*}$ equations (4.7) for the chiral primaries with

\footnotetext{
${ }^{16}$ Notice that under this automorphism the superconformal partners rotate as $S \rightarrow e^{-i \theta} S, \bar{S} \rightarrow e^{i \theta} \bar{S}$.
} 
$R=4$ and that of $\mathcal{L}^{4}$ is four times that of $\mathcal{L}$ given in (6.2). Putting everything together we find the following equation for the Riemann tensor on the moduli space

$$
\frac{1}{(2 \pi)^{4}} R_{i \bar{j} k}^{l}=-C_{i k}^{M} g_{M \bar{N}} C_{\bar{j} \bar{q}}^{* \bar{N}} g^{\bar{q} l}+g_{k \bar{j}} \delta_{i}^{l}+g_{i \bar{j}} \delta_{k}^{l}-\frac{8}{3 c} g_{i \bar{j}} \delta_{k}^{l}
$$

where $C_{i j}^{M}$ are the chiral ring coefficients between chiral primaries $\Phi_{i}, \Phi_{j}$ of $\Delta=2$ and $\phi_{M}$ of $\Delta=4$ and $g_{i \bar{j}}$ is proportional to the metric on the moduli space $G_{i \bar{j}}$ (see (3.3)).

These are partial differential equations for the Zamolodchikov metric $G_{i \bar{j}}$ on $\mathcal{M}$, with coefficients given by the holomorphic correlators $C_{i j}^{M}$ and their complex conjugates. These equations are generalizations of special geometry and similar to those describing the WeilPetersson metric on the moduli space of Calabi-Yau n-folds or those of $\mathcal{N}=(2,2)$ SCFTs in two dimensions with arbitrary central charge [32-36].

\section{$7 \mathcal{N}=1$ theories}

Let us now explain which of our previous results are true for theories with $\mathcal{N}=1$ superconformal invariance in four dimensions. The $\mathcal{N}=1$ SCFT has a $\mathrm{U}(1)_{\mathrm{R}} \mathrm{R}$-symmetry. The standard normalization of the $\mathrm{U}(1)_{\mathrm{R}}$ in $\mathcal{N}=1$ theories is such that the unitarity bound is $\Delta \geq \frac{3}{2} R$. Superconformal primary operators saturating this bound are chiral primaries and are annihilated by the supercharges of right chirality $\bar{Q}_{\dot{a}}$. We have the structure of a chiral ring as in $\mathcal{N}=2$ theories and we use the same notation for the 2- and 3-point functions $g_{i \bar{j}}$ and $C_{i j \bar{k}}$ and the chiral ring coefficients $C_{i j}^{k}$.

In $\mathcal{N}=1$ SCFTs marginal deformations preserving supersymmetry are descendants of chiral primaries $\Phi_{i}$ of $\Delta=3$ and again they split into holomorphic and antiholomorphic ones

$$
\mathcal{O}_{i}=Q^{2} \cdot \Phi_{i} \quad, \quad \overline{\mathcal{O}}_{j}=\bar{Q}^{2} \cdot \bar{\Phi}_{j}
$$

All of the results of section 4.1 are also true for $\mathcal{N}=1$ theories. In particular the 3 -point functions of chiral primaries satisfy the following set of equations

$$
\begin{aligned}
\bar{\nabla}_{j} C_{k} & =0, & \nabla_{i} \bar{C}_{k} & =0 \\
\nabla_{i} C_{j} & =\nabla_{j} C_{i}, & \bar{\nabla}_{i} \bar{C}_{j} & =\bar{\nabla}_{j} \bar{C}_{i} \\
{\left[C_{i}, C_{j}\right] } & =0, & {\left[\bar{C}_{i}, \bar{C}_{j}\right] } & =0
\end{aligned}
$$

where the notation $C_{i}$ means that we think of it as a matrix $\left(C_{i}\right)_{k}^{l}$. We can also show that the following relations are true

$$
\begin{aligned}
& {\left[\nabla_{i}, \nabla_{j}\right]_{k}^{l}=0} \\
& {\left[\bar{\nabla}_{i}, \bar{\nabla}_{j}\right]_{k}^{l}=0}
\end{aligned}
$$

The last equation implies that we can pick a holomorphic gauge as before and then we have the following equations

$$
\begin{aligned}
\partial_{\bar{m}} C_{i j}^{k} & =0 \\
\partial_{i} C_{j k}^{l}-\partial_{j} C_{i k}^{l} & =\left[g^{-1} \partial_{i} g, C_{j}\right]_{k}^{l}-\left[g^{-1} \partial_{j} g, C_{i}\right]_{k}^{l}
\end{aligned}
$$


We can also show that "extremal" correlators have to be holomorphic

$$
\bar{\nabla}_{m}\left\langle\phi_{1}\left(x_{1}\right) \ldots \phi_{n}\left(x_{n}\right) \bar{\phi}_{n+1}(z)\right\rangle=0
$$

It would be very interesting to see whether the third of the $t t^{*}$ equations (4.7) can also be derived for $\mathcal{N}=1$ SCFTs, possibly with some modifications. We hope to report on this in the future [19].

\section{$8 \mathcal{N}=4$ theories}

Now we discuss how the previous analysis applies to theories with $\mathcal{N}=4$ superconformal invariance. Certain aspects of the connection on the space of operators for $\mathcal{N}=4$ theories have been discussed in [37, 38]. An $\mathcal{N}=4$ theory can also be viewed as an $\mathcal{N}=2$ theory and has several kinds of short supermultiplets [20,21]. Superconformal multiplets of the $\mathcal{N}=4$ algebra are labeled by the conformal dimension $\Delta$, the $\operatorname{spin}(j, \bar{j})$ and by their $\mathrm{SO}(6)_{\mathrm{R}} \mathrm{R}$-symmetry representation.

\subsection{The moduli space for $\mathcal{N}=4$ gauge theories}

All examples of $\mathcal{N}=4$ superconformal field theories that we know are realized as Lagrangian gauge theories with a product gauge group $\prod_{i=1}^{n} G_{i}$, each with a coupling constant $\tau_{i}$. Then the moduli space is the direct product of the individual moduli spaces, at least locally. So for simplicity we can focus on the case where we only have a single gauge group.

The marginal operator in that case is a descendant of fields in a short multiplet whose superconformal primaries are scalar fields $\Phi$ with conformal dimension $\Delta=2$ and transforming into the rank two symmetric traceless representation of $\mathrm{SO}(6) \cdot{ }^{17}$ It is not hard to show that the $\mathrm{SO}(6)_{\mathrm{R}}$ quantum numbers of fields do not change under motion on the moduli space. So the holonomy on the multiplet $\Phi$ must commute with $\mathrm{SO}(6)$ which implies that the holonomy is actually trivial. So the multiplet $\Phi$ does not receive any holonomy under motion on the moduli space.

This means that the curvature of the tangent bundle is simply given by the curvature of the bundle $\mathcal{L}$ of the supercharges ${ }^{18}$ which being proportional to the Kähler form, is covariantly constant. Hence the moduli space is locally the homogeneous hyperbolic ${ }^{19}$ space

$$
\frac{\mathrm{SO}(1,2)}{\mathrm{SO}(2)}
$$

It is standard to parametrize it in terms of the complexified gauge coupling

$$
\tau=\frac{\theta}{2 \pi}+i \frac{4 \pi}{g_{Y M}^{2}}
$$

\footnotetext{
${ }^{17}$ The gauge invariant operators $\Phi$ should not be confused with the adjoint-valued elementary scalar fields $X_{i} i=1, \ldots 6$ of the $\mathcal{N}=4$. Schematically we have $\Phi \sim \operatorname{Tr}\left(X_{(i} X_{j)}\right)$, where we did not write down the $\mathrm{SO}(6)_{\mathrm{R}}$ indices of $\Phi$ and parentheses around the indices denote symmetrization and subtraction of the trace.

${ }^{18}$ Similar to the $\mathcal{N}=2$ superconformal algebra the $\mathcal{N}=4$ algebra has an outer automorphism under which $Q_{a}^{i} \rightarrow e^{i \theta} Q_{a}^{i}, \bar{Q}_{i, \dot{a}} \rightarrow e^{-i \theta} \bar{Q}_{i, \dot{a}}$.

${ }^{19}$ From (6.2) we can see that the scalar curvature is negative.
} 
with the metric

$$
d s^{2} \sim c \frac{d \tau d \bar{\tau}}{\operatorname{Im} \tau^{2}}
$$

The global structure depends on the action of the S-duality group and cannot be determined by our local analysis. We see from (6.2) that the overall scale of the metric is proportional to the central charge $c$. This can be independently checked by a weak coupling computation of the Zamolodchikov metric in the $\mathcal{N}=4$ SYM. In the case of the $\mathcal{N}=4 \mathrm{SYM}$ with gauge group $\mathrm{SU}(\mathrm{N})$ this metric agrees in the large $N$ limit with the metric on the moduli space of the axion-dilaton of IIB supergravity in $A d S_{5} \times S^{5}$.

If we have $n$ gauge group factors we will have $n$ corresponding short multiplets $\Phi_{i}$ which give marginal operators and then the moduli space is (locally) the product of spaces of the form (8.1). The statement that the gauge group is a direct product is related to the fact that the $\Phi_{i}$ 's do not mix among themselves under motion on the moduli space.

\subsection{Exotic $\mathcal{N}=4$ theories?}

In this speculative paragraph let us mention that it is a logical possibility that exotic $\mathcal{N}=4$ superconformal field theories may exist, not realizable as weakly coupled Lagrangian theories anywhere on their moduli space, where the space of coupling constants is not just a direct product of "gauge couplings". That would correspond to an $\mathcal{N}=4$ theory with $n$ short supermultiplets of $\Delta=2$, which contain marginal operators in their descendants, and which do mix nontrivially under motion on the moduli space. How complicated can this mixing be? Since the mixing has to commute with the $\mathrm{SO}(6) \mathrm{R}$-symmetry we conclude that it can be at most an $\mathrm{SO}(\mathrm{n})$ transformation.

The moduli space of such a theory would have real dimension $2 n$. The most general holonomy of a 2 n-dimensional manifold is $\mathrm{SO}(2 \mathrm{n})$. On the other hand we know that the holonomy of the tangent bundle is the product of the $\mathrm{SO}(2)$ holonomy of the supercharges with the $\mathrm{SO}(\mathrm{n})$ of the $n$ short multiplets, so $\mathrm{SO}(2) \otimes \mathrm{SO}(\mathrm{n})$ in total. Then the moduli space is a manifold of reduced holonomy. Berger's classification of manifolds with reduced holonomy then fixes the metric on the moduli space and we learn that the moduli space is locally of the form

$$
\frac{\mathrm{SO}(\mathrm{n}, 2)}{\mathrm{SO}(\mathrm{n}) \otimes \mathrm{SO}(2)}
$$

This is the most general moduli space consistent with $\mathcal{N}=4$ superconformal invariance in four dimensions. ${ }^{20}$ As far as we know, there are no indications for the existence of any exotic $\mathcal{N}=4$ theory of this type.

\subsection{Chiral primaries for $\mathcal{N}=4$ theories}

For chiral primaries of the $\mathcal{N}=4$ one can show that the chiral ring coefficients are covariantly constant along all directions of the moduli space. Then taking the covariant derivatives of both sides of the $t t^{*}$ equations we get that the curvature of the bundles of

\footnotetext{
${ }^{20}$ The argument we followed is analogous to the one in [39] generalizing the results of [26] for the moduli space of two-dimensional $\mathcal{N}=(4,4)$ theories.
} 
chiral primaries are covariantly constant

$$
\nabla F=0
$$

In the previous section we found that the moduli space of $\mathcal{N}=4$ theories is a homogeneous space. Bundles of covariantly constant curvature over homogeneous spaces are called homogeneous bundles and their structure is completely determined by group theory [40], in analogy with the two-dimensional story in [18]. For Lagrangian $\mathcal{N}=4$ theories this implies that the bundles of chiral primaries are (locally) flat. For "exotic" $\mathcal{N}=4$ theories, if they exist, we would have nontrivial homogeneous bundles labeled by representations of $\mathrm{SO}(\mathrm{n})$. These issues will be discussed in more detail in [19].

\section{A comment on the moduli space of vacua}

In this paper the term "moduli space" has been used to refer to the parameter space of the conformal field theory. This has to be distinguished from the moduli space of vacua. The first parametrizes a family of conformal field theories which are continuously connected, while the second is a family of vacua of a given and fixed conformal field theory. The first is a characteristic of the quantum field theory in the UV and refers to a tuning of the parameters in its Lagrangian, while the second is an IR concept, which is characterized by vacuum expectation values of certain operators.

Notice that in two-dimensional conformal field theory this distinction is not usually made because in two dimensions there cannot be moduli spaces of vacua due to infrared divergences. The situation is different in higher dimensional conformal field theories where it is possible for operators to get nonzero vevs.

It is also natural to consider the total space, the space of all possible vacua of all continuously connected conformal field theories. This has the structure of a bundle where the base is the moduli space of conformal field theories and the fiber is the space of vacua for the given theory [41]. Perhaps it would be interesting to explore the topology and geometry of this total space in more detail.

In many Lagrangian $\mathcal{N}=2$ gauge theories this total space is nicely described by the Seiberg-Witten curves [1, 42], which in the case of finite theories depend on the marginal coupling $\tau$ and the vevs of the gauge invariant operators which parametrize the position on the Coulomb branch. In Lagrangian theories we look for vacua by minimizing a potential and computing the vevs of gauge invariant operators on the space of solutions. To understand the similar structure for more general superconformal field theories, without Lagrangian description, it would be necessary to work with the concept of a "vacuum" in more abstract conformal field theory language. In this framework we imagine that we are only given the list of the primary (gauge invariant) operators $\mathcal{O}_{i}$ and their conformal dimensions $\Delta_{i}$, as well as the 3-point functions $C_{i j}^{k}$. In a nontrivial (i.e. non-conformaly invariant) vacuum the primaries can get nonzero vevs $\left\langle\mathcal{O}_{i}\right\rangle=A_{i} M^{\Delta_{i}}$, where $A_{i}$ are dimensionless numbers and $M$ is a mass scale which characterizes the breaking of the scale invariance in the vacuum. Only some combinations of vevs are allowed by the dynamics of the theory and the 
set of such allowed combinations $\left\{A_{i}\right\}$ is the moduli space of vacua of the CFT. It would be interesting to formulate these conditions in terms of the CFT data $\Delta_{i}$ and $C_{i j}^{k}$.

\section{Discussions}

We derived some new exact results for the chiral ring of general $\mathcal{N}=2$ superconformal field theories and found structures similar to those encountered in two-dimensional $\mathcal{N}=(2,2)$ theories. Our analysis was based on conformal perturbation theory. It would be interesting to study whether our equations can be derived in an alternative way by considering the topologically twisted $\mathcal{N}=2$ theory [43] and following similar arguments as in [17]. Such an approach might also give results about massive deformations of $\mathcal{N}=2$ superconformal field theories, which could be useful for applications to theories with less supersymmetry. It might also be interesting to explore the topologically twisted $\mathcal{N}=2$ superconformal field theories on more general four-manifolds. For such an approach the analysis of [44] might be useful and also [45] for recent discussions.

It would be instructive to apply our somewhat abstract analysis to specific examples, such as the finite $\mathcal{N}=2 \mathrm{SU}(\mathrm{N})$ gauge theories with $N_{f}=2 N$ flavors in the fundamental. In these theories the chiral ring is generated by chiral primaries of the form $\operatorname{Tr}\left(\Phi^{k}\right)$ where $\Phi$ is the adjoint scalar field and the moduli space is parametrized by the gauge coupling constant $\tau=\frac{\theta}{2 \pi}+i \frac{4 \pi}{g_{Y M}^{2}}$. It is possible to perform perturbative computations in the $\tau \rightarrow i \infty$ limit and then we can use the holomorphy of the chiral ring coefficients and the $t t^{*}$ equations to extend the computation in the interior of the moduli space. In particular it would be interesting to try to connect the $\tau \rightarrow i \infty$ point with the S-dual "infinitely-strongly coupled" limit $\tau \rightarrow 1$ discussed in [46].

More generally it would be interesting to analyze the rich class of $\mathcal{N}=2$ superconformal field theories studied by Gaiotto in [13]. These theories have more complicated moduli spaces related to those of punctured Riemann surfaces. Based on this, it was pointed out in [47] that the Zamolodchikov metric on the moduli spaces of such conformal field theories is the Weil-Petersson metric on the Teichmuller space, which is consistent with the equations that we found in section 6 . The chiral primary operators of the four-dimensional theory are related to holomorphic differentials on the Riemann surface on which the M5 branes are wrapped. Thus the connection for the chiral primaries that we computed is related to the Gauss-Manin connection for the cohomology elements of the Riemann surface under variation of its complex structure moduli. It would be nice to make these statements more precise.

It might also be useful to explore our results from the six-dimensional point of view. A six-dimensional superconformal field theory can be either wrapped on a Riemann surface giving an $\mathcal{N}=2$ theory in four dimensions, or on a 4-manifold resulting into a SCFT in 2 dimensions. Both theories obey the $t t^{*}$ equations and it would be nice to understand relations between the two and possible connections with [48, 49].

Another interesting direction is clarifying the bulk interpretation of our results in cases of $\mathcal{N}=2$ theories with AdS duals. The moduli space of the conformal field theory 
is mapped to the moduli space of the AdS compactification of supergravity/string theory. The chiral primaries are related to certain Kaluza-Klein modes in the internal manifold, or possibly other supersymmetric probes such as giant gravitons.

It is worth trying to extend our results in $\mathcal{N}=1$ superconformal field theories in four dimensions. Upon dimensional reduction on a two-torus they lead to two-dimensional $\mathcal{N}=(2,2)$ supersymmetric theories (not necessarily conformal). Hence one might hope that some degree of control exists even in the parent four-dimensional theory, see also [50]. From the AdS/CFT point of view the structure of the moduli space of $\mathcal{N}=1$ superconformal field theories was briefly discussed in [51] in relation to a-maximization [52]. It might be possible to explore these questions using methods similar to the ones developed in this paper.

Our analysis has been local on the moduli space. It would be interesting to analyze certain global issues such as the action of the S-duality group on correlators and possible monodromies for the chiral primaries around non-contractible cycles. Of interest would also be to analyze what happens at points where chiral primaries combine into long multiplets and lift [53]. In a sense these seem like the SCFT analogue ${ }^{21}$ of "walls of marginal stability".

It would be nice to check that the renormalization method that we adopted to implement conformal perturbation theory is actually consistent with all axioms of conformal field theory and with supersymmetry, especially at higher orders in the perturbation.

Finally let us mention that the connection of operators that we have computed can be interpreted as Berry's phase [57] (and its non-abelian generalization [58]) for a class of states as we now explain. If we define the conformal field theory on $\mathbf{S}^{\mathbf{3}} \times$ time then it can be thought of as a quantum system whose Hilbert space is isomorphic to the set of local operators on the plane, via the state-operator map. These states fall into unitary representations of the superconformal algebra. A special class of these states, the ones that are dual to the chiral primary operators, belong to shorter representations. If we adiabatically vary the coupling constants of the theory, then states are subject to the phenomenon known as geometric or Berry's phase. The $t t^{*}$ equations compute the Berry phase for the chiral states. In the AdS bulk this would be the Berry phase of certain quantum states under adiabatic variation of the supergravity moduli. See also [59] and [60-63].

\section{Acknowledgments}

We would like to thank L. Hollands, E. Kiritsis, M. Shigemori, M. Taylor, D. Tong, C. Vafa, E. Witten and especially J. de Boer, K. Skenderis and E. Verlinde for useful discussions and comments on the manuscript. We thank the University of Crete for hospitality during the completion of this work.

\footnotetext{
${ }^{21}$ Here we are talking about joining and splitting of BPS multiplets of the superconformal group, i.e. of local operators, as a function of the position on the moduli space of the CFT and not about the joining and splitting of BPS dyons as a function of the position on the moduli space of vacua. Unlike what happens for BPS dyons, the joining and splitting of BPS local operators happens along surfaces of codimension larger than one, so the term "wall" in this case may not be so accurate. It would be useful to understand the rules of joining and splitting of such BPS states and possible relations with [54-56].
} 


\section{A Superconformal Ward identities}

We will show how to derive the Ward identities (2.1) and (2.3) in more detail. As a warmup we review the derivation of the Ward identity for a conserved bosonic current. Of course this is a standard result, but we want to derive it from a more abstract conformal field theory point of view, without any reference to an action or the Noether procedure. Our starting point is that the CFT has a spin $(1 / 2,1 / 2)$ operator $J^{\mu}$ of $\Delta=3$. Using the conformal algebra we can compute the norm of the first descendant $\partial_{\mu} J^{\mu}$ and we find that it vanishes, which implies the operator equation $\partial_{\mu} J^{\mu}=0$, so $J^{\mu}$ is a conserved current. We define the corresponding conserved charge by $R=\int d^{3} x J^{0}$. For any local operator $\varphi$ we have the following $\mathrm{OPE}$

$$
J^{\mu}(x) \varphi(0)=\ldots+\frac{1}{2 \pi^{2}} \frac{x^{\mu}}{|x|^{4}}[R, \varphi](0)+\ldots
$$

with no other terms of the form $\frac{x^{\mu}}{|x|^{4}}$. This can be written as

$$
[R, \varphi](x)=\oint_{x} d S_{\mu} \cdot J^{\mu}(z) \varphi(x)
$$

where we compute the integral over a small sphere ${ }^{22}$ surrounding the point $x$. Now consider the general correlator

$$
V^{\mu}(z)=\left\langle J^{\mu}(z) \varphi_{1}\left(x_{1}\right) \ldots \varphi_{n}\left(x_{n}\right)\right\rangle
$$

where we assume that all points are distinct. If we keep $x_{k}$ fixed and think of $V$ as a function of $z$ then it is vector field defined on $\mathcal{D}=\mathbf{R}^{4}-\left\{x_{1}, \ldots, x_{n}\right\}$, which is divergenceless $\partial_{\mu} V^{\mu}=0$, since $\partial_{\mu} J^{\mu}=0$. Then

$$
0=\int_{\mathcal{D}} \partial_{\mu} V^{\mu}=\int_{\partial \mathcal{D}} d S_{\mu} \cdot V^{\mu}=\oint_{\infty} d S_{\mu} \cdot V^{\mu}(z)-\sum_{i} \oint_{x_{i}} d S_{\mu} \cdot V^{\mu}(z)
$$

Since $J^{\mu}$ has conformal dimension $\Delta=3$ we know that $V^{\mu}(z)$ falls off at least as $\frac{1}{|z|^{6}}$ at large $z$, so there is no contribution from the sphere at infinity in (A.2) and we only get contributions from the punctures $\left\{x_{1}, \ldots, x_{n}\right\}$. Using (A.1) we find

$$
\sum_{k}\left\langle\varphi_{1}\left(x_{1}\right) \ldots\left[R, \varphi_{k}\right]\left(x_{k}\right) \ldots \varphi_{n}\left(x_{n}\right)\right\rangle=0
$$

which is the desired Ward identity expressing charge conservation.

Now we will do the same thing for the supercurrent. The left chiral supercurrent $G_{a}^{i \mu}$ is a conformal primary operator of dimension $\Delta=7 / 2$ transforming in the $(1,1 / 2)$ representation of the Lorentz group (indices $a, \mu$ ) and in the $I=1 / 2$ of $\mathrm{SU}(2)_{\mathrm{R}}$ (index $i$ ). It satisfies the following equations

$$
\partial_{\mu} G_{a}^{i \mu}=0, \quad G_{a}^{i \mu} \sigma_{\mu}^{\dot{a} a}=0
$$

\footnotetext{
${ }^{22}$ We compute the integral on a sphere of radius $\epsilon$ and then take the limit $\epsilon \rightarrow 0$.
} 
We can construct conserved fermionic currents by multiplying the supercurrent with a conformal killing spinor $\psi^{a}(x)$ as

$$
j^{i \mu}(x)=\psi^{a}(x) G_{a}^{i \mu}(x)
$$

It is easy to show that

$$
\partial_{\mu} j^{i \mu}=0
$$

and the corresponding conserved supercharge is $\int d^{3} x j^{i 0}$. In $\mathbf{R}^{4}$ the most general conformal killing spinor is

$$
\psi^{a}(x)=\lambda^{a}+x^{\dot{a} a} \mu_{\dot{a}}
$$

where $\lambda^{a}$ is an arbitrary constant $(1 / 2,0)$ spinor, $\mu_{\dot{a}}$ an arbitrary constant $(0,1 / 2)$ spinor and we use the notation $x^{\dot{a} a}=x^{\mu} \sigma_{\mu}^{\dot{a} a}$. If we take $\lambda^{a} \neq 0$ and $\mu_{\dot{a}}=0$ we generate the left chiral supercharges $Q_{a}^{i}$, while for $\lambda^{a}=0$ and $\mu_{\dot{a}} \neq 0$ we get the right chiral superconformal charges $\bar{S}^{i \dot{a}}$

$$
Q_{a}^{i} \sim \int d^{3} x G_{a 0}^{i}, \quad \bar{S}^{i \dot{a}} \sim \int d^{3} x x^{\dot{a} a} G_{a 0}^{i}
$$

In a similar way the right chiral supercurrent $\bar{G}_{i \dot{a}}^{\mu}$ generates the right chiral supercharges $\bar{Q}_{i \dot{a}}$ and the left chiral superconformal charges $S_{i}^{a}$.

The OPE of the supercurrent with a scalar primary operator $\varphi$ has the following form

$$
G_{a}^{i \mu}(x) \varphi(0)=\ldots+\frac{x^{\mu} x_{a \dot{b}}}{2 \pi^{2}|x|^{6}}\left\{\bar{S}^{i \dot{b}}, \varphi\right\}(0)+\frac{x^{\mu}}{2 \pi^{2}|x|^{4}}\left\{Q_{a}^{i}, \varphi\right\}(0)+\ldots
$$

For every choice of the conformal Killing spinor (A.5) we can derive a Ward identity for the corresponding conserved current $j^{i \mu}$ given in (A.4). We start with

$$
\left\langle j^{i \mu}(z) \varphi_{1}\left(x_{1}\right) \ldots \varphi_{n}\left(x_{n}\right)\right\rangle
$$

and follow the same steps as before ${ }^{23}$. Being careful about the expansion of $j^{i \mu}$ around the punctures and using (A.6) we find the following superconformal Ward identity

$$
\begin{aligned}
\sum_{k=1}^{n} \psi^{a}\left(x_{k}\right)\left\langle\varphi_{1}\left(x_{1}\right) \ldots\right. & \left.\left\{Q_{a}^{i}, \varphi_{k}\right\}\left(x_{k}\right) \ldots \varphi_{n}\left(x_{n}\right)\right\rangle \\
& -\sum_{k=1}^{n}\left(\partial_{\mu} \psi^{a}\right) \sigma_{a \dot{b}}^{\mu}\left(x_{k}\right)\left\langle\varphi_{1}\left(x_{1}\right) \ldots\left\{\bar{S}^{i b}, \varphi_{k}\right\}\left(x_{k}\right) \ldots \varphi\left(x_{n}\right)\right\rangle=0
\end{aligned}
$$

If we take $\psi^{a}(x)$ to be constant then we find that the usual supercharges can be moved around without any factors

$$
\sum_{k=1}^{n}\left\langle\varphi_{1}\left(x_{1}\right) \ldots\left\{Q_{a}^{i}, \varphi_{k}\right\}\left(x_{k}\right) \ldots \varphi_{n}\left(x_{n}\right)\right\rangle=0
$$

\footnotetext{
${ }^{23}$ We also have to use that $G_{a}^{i \mu}$ has $\Delta=7 / 2$, so if it is taken to infinity inside any correlator, it falls off at least as $\frac{1}{|z|^{7}}$.
} 
and if we take $\psi^{a}(x)$ to be proportional to $x$ we find that for the superconformal partners we have

$$
\sum_{k=1}^{n}\left\langle\varphi_{1}\left(x_{1}\right) \ldots\left\{\left(x_{k}-x_{0}\right)^{\dot{a} a} Q_{a}^{i}-\bar{S}^{i, \dot{a}}, \varphi_{k}\right\}\left(x_{k}\right) \ldots \varphi_{n}\left(x_{n}\right)\right\rangle=0
$$

for any $x_{0}$.

From (A.7) we see that if a field $\varphi$ is a superconformal primary (hence annihilated by the $\bar{S}$ 's) then we can choose the conformal Killing spinor $\psi^{a}(x)$ to vanish at the point of insertion of $\varphi$ and that when applying the superconformal Ward identity (for this special $\left.\psi^{a}(x)\right)$ the field $\varphi$ does not contribute at all, whether it is annihilated by the $Q$ 's or not. This is the essence of identity (2.3). In two dimensions it was discussed in [14, 64].

For completeness we present the Ward identities for the right chiral supercurrent $\bar{G}_{i \dot{a}}^{\mu}$

$$
\begin{aligned}
\sum_{k=1}^{n} \psi^{\dot{a}}\left(x_{k}\right)\left\langle\varphi_{1}\left(x_{1}\right) \ldots\right. & \left.\left\{\bar{Q}_{i \dot{a}}, \varphi_{k}\right\}\left(x_{k}\right) \ldots \varphi_{n}\left(x_{n}\right)\right\rangle \\
& +\sum_{k=1}^{n}\left(\partial_{\mu} \psi^{\dot{a}}\right) \sigma_{b \dot{a}}^{\mu}\left(x_{k}\right)\left\langle\varphi_{1}\left(x_{1}\right) \ldots\left\{S^{b}, \varphi_{k}\right\}\left(x_{k}\right) \ldots \varphi_{n}\left(x_{n}\right)\right\rangle=0
\end{aligned}
$$

which implies

$$
\sum_{k=1}^{n}\left\langle\varphi_{1}\left(x_{1}\right) \ldots\left\{\bar{Q}_{i, \dot{a}}, \varphi_{k}\right\}\left(x_{k}\right) \ldots \varphi_{n}\left(x_{n}\right)\right\rangle=0
$$

and

$$
\sum_{k=1}^{n}\left\langle\varphi_{1}\left(x_{1}\right) \ldots\left\{\left(x_{k}-x_{0}\right)^{\dot{a} a} \bar{Q}_{i, \dot{a}}+S_{i}^{a}, \varphi_{k}\right\}\left(x_{k}\right) \ldots \varphi_{n}\left(x_{n}\right)\right\rangle=0
$$

for all $x_{0}$.

\section{B Some basic properties of $\mathcal{N}=2$ superconformal theories}

The supermultiplet containing the stress-energy tensor and the other conserved currents begins with a scalar superconformal primary operator $\mathcal{A}$ with $\Delta=2, I=0, R=0$. It is a short multiplet of the superconformal algebra [65]. The other conformal primaries in the multiplet are (diagram is from Dolan and Osborn [66], with slightly different conventions for the $R$-charge)

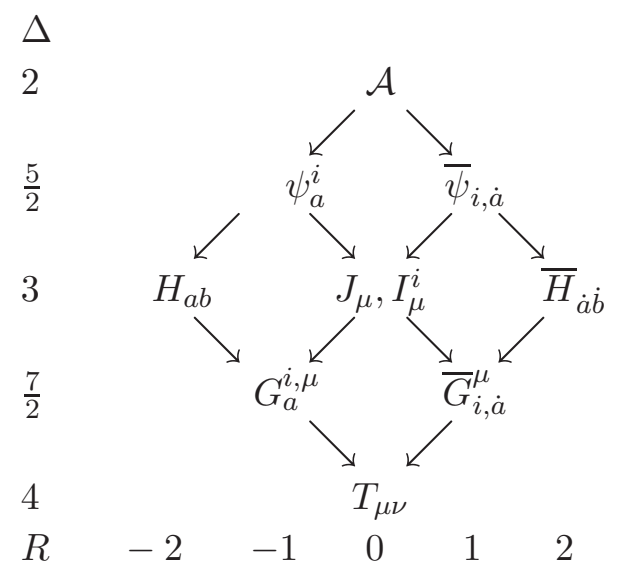


where the $\swarrow$ arrows denote the action of the left-chiral supercharges $Q_{a}^{i}$ and $\searrow$ of $\bar{Q}_{i, \dot{a}}$. The conformal dimension $\Delta$ and $\mathrm{U}(1)_{\mathrm{R}}$ charge $R$ of the operators can be seen in the diagram. The fermions $\psi_{a}^{i}$ have spin $(1 / 2,0)$ and transform in the $I=1 / 2$ representation of $\mathrm{SU}(2)_{\mathrm{R}}$. The $\mathrm{U}(1)_{\mathrm{R}}$ current is $J_{\mu}$ and the $\mathrm{SU}(2)_{\mathrm{R}}$ currents are $I_{\mu}^{i}, i=1,2,3$. The field $H_{a b}$ is a spin $(1,0)$ operator singlet under the $\mathrm{SU}(2)_{\mathrm{R}}$. The fields $G_{a}^{i, \mu}$ (and their conjugates) are the supercurrents and transform in the $I=1 / 2$ of $\mathrm{SU}(2)_{\mathrm{R}}$. The stress-energy tensor $T_{\mu \nu}$ has spin $(1,1), I=0$ and $R=0$.

$\mathcal{N}=2$ SCFTs have two "central charges" $c, a$ defined as coefficients of the trace anomaly on a curved manifold

$$
\left\langle T_{\mu}^{\mu}\right\rangle=\frac{c}{16 \pi^{2}}(\text { Weyl })^{2}-\frac{a}{16 \pi^{2}}(\text { Euler })
$$

The 2-point functions of fields in the supermultiplet of $\mathcal{A}$ have the form

$$
\begin{aligned}
\langle\mathcal{A}(x) \mathcal{A}(0)\rangle & =\frac{3 c}{8 \pi^{4}} \frac{1}{|x|^{4}} \\
\left\langle J_{\mu}(x) J_{\nu}(0)\right\rangle & =\frac{12 c}{\pi^{4}} \frac{I_{\mu \nu}(x)}{|x|^{6}} \\
\left\langle T_{\mu \nu}(x) T_{\rho \sigma}(y)\right\rangle & =\frac{40 c}{\pi^{4}} \frac{\mathcal{I}_{\mu \nu, \rho \sigma}(x)}{|x|^{8}}
\end{aligned}
$$

where

$$
\begin{aligned}
I_{\mu \nu}(x) & \equiv g_{\mu \nu}-2 \frac{x_{\mu} x_{\nu}}{|x|^{2}} \\
\mathcal{I}_{\mu \nu, \rho \sigma} & =\frac{1}{2}\left(I_{\mu \sigma}(x) I_{\nu \rho}(x)+I_{\mu \rho}(x) I_{\nu \sigma}(x)-\frac{1}{4} \delta_{\mu \nu} \delta_{\rho \sigma}\right)
\end{aligned}
$$

We also have the 3 -point function [67]

$$
\left\langle\mathcal{A}\left(x_{1}\right) \mathcal{A}\left(x_{2}\right) \mathcal{A}\left(x_{3}\right)\right\rangle=\frac{3(4 a-3 c)}{32 \pi^{6}} \frac{1}{\left|x_{12}\right|^{2}\left|x_{23}\right|^{2}\left|x_{13}\right|^{2}}
$$

We proceed with a few more basic results that we need. Starting with the $U(1)_{R}$ currents $J^{\mu}$, we define the corresponding conserved charge by $\int d^{3} x J^{0}(x)$. If a scalar primary operator $\mathcal{O}$ has charge $R$ under the $\mathrm{U}(1)_{\mathrm{R}}$ then we have an OPE of $\mathcal{O}$ with $J_{\mu}$ of the form

$$
J_{\mu}(x) \mathcal{O}(0)=\ldots+\frac{R}{2 \pi^{2}} \frac{x^{\mu}}{|x|^{4}} \mathcal{O}(0)+\ldots
$$

If the 2-point function of the operator is normalized as

$$
\langle\mathcal{O}(x) \overline{\mathcal{O}}(0)\rangle=\frac{g_{\mathcal{O} \overline{\mathcal{O}}}}{|x|^{2 \Delta}}
$$

then the 3-point function with the $\mathrm{U}(1)_{\mathrm{R}}$ current is

$$
\left\langle\mathcal{O}\left(x_{1}\right) \overline{\mathcal{O}}\left(x_{2}\right) J_{\mu}\left(x_{3}\right)\right\rangle=-g_{\mathcal{O}} \frac{R}{2 \pi^{2}} \frac{1}{\left|x_{12}\right|^{2 \Delta-2}\left|x_{13}\right|^{2}\left|x_{23}\right|^{2}}\left(\frac{\left(x_{13}\right)_{\mu}}{\left|x_{13}\right|^{2}}-\frac{\left(x_{23}\right)_{\mu}}{\left|x_{23}\right|^{2}}\right)
$$


and we also have

$$
\mathcal{O}(x) \overline{\mathcal{O}}(0)=\ldots-g_{\mathcal{O} \overline{\mathcal{O}}} R \frac{\pi^{2}}{24 c} \frac{x^{\mu}}{|x|^{2 \Delta-2}} J_{\mu}(0)+\ldots
$$

Then for chiral primaries $\phi_{i}, \phi_{j}$ of the same $\mathrm{U}(1)_{\mathrm{R}}$ charge $q$ we have the following OPE coefficients with the $\mathrm{U}(1)_{\mathrm{R}}$ current

$$
C_{i \bar{j}}^{J}=-g_{i \bar{j}} R \frac{\pi^{2}}{24 c}
$$

We will also need the OPE coefficient $C_{i \bar{j}}^{\mathcal{A}}$. As we now show it is also fixed by the Ward identities. First we write $J_{\mu}=\lambda \sigma_{\mu}^{\dot{b} a}\left[\left[Q_{a}^{i}, \bar{Q}_{i, b}\right], \mathcal{A}\right]$. The constant $\lambda$ can be fixed by demanding consistency with the 2-point functions (B.2). After some algebra we find that $\lambda=\frac{1}{4 \sqrt{2}}$. Then using the superconformal Ward identities we find

$$
\left\langle\phi_{i}\left(x_{1}\right) \bar{\phi}_{j}\left(x_{2}\right) J_{\mu}\left(x_{3}\right)\right\rangle=\frac{4}{\sqrt{2}}\left\langle\phi_{i}\left(x_{1}\right) \bar{\phi}_{j}\left(x_{2}\right) \mathcal{A}\left(x_{3}\right)\right\rangle\left(\frac{\left(x_{13}\right)_{\mu}}{\left|x_{13}\right|^{2}}-\frac{\left(x_{23}\right)_{\mu}}{\left|x_{23}\right|^{2}}\right)
$$

Then comparing with (B.5) and using (B.2) we find

$$
C_{i \bar{j} \mathcal{A}}=-g_{i \bar{j}} R \frac{\sqrt{2}}{8 \pi^{2}}, \quad C_{i \bar{j}}^{\mathcal{A}}=-\frac{\sqrt{2}}{3 c} \pi^{2} R g_{i \bar{j}}
$$

If we also have other chiral primaries $\phi_{k}, \phi_{l}$ of $\mathrm{R}$-charge $R^{\prime}$ then we have

$$
C_{i \bar{j}}^{\mathcal{A}} C_{\mathcal{A l}, k}=\frac{R R^{\prime}}{12 c} g_{i \bar{j}} g_{k \bar{l}}
$$

\section{Contours}

Now let us consider the following quantity

$$
\begin{aligned}
\mathcal{E}= & \lim _{r \rightarrow 1^{-}} \int_{|x|=r} d \Omega_{3}^{x} \int_{|y|=1} d \Omega_{3}^{y}|x|^{2}|y|^{2}\left(y \cdot \partial_{y}\right)\left(x \cdot \partial_{x}\right) \\
& \times\left(\frac{|y|^{2}}{|x|^{2}}\left\langle\bar{\phi}_{l}(\infty) \Phi_{i}(x) \bar{\Phi}_{j}(y) \phi_{k}(0)\right\rangle-\frac{|x|^{2}}{|y|^{2}}\left\langle\bar{\phi}_{l}(\infty) \Phi_{i}(y) \bar{\Phi}_{j}(x) \phi_{k}(0)\right\rangle\right)
\end{aligned}
$$

In this double integral the variable $y$ lies on a 3 -sphere of unit radius while $x$ on a 3 -sphere of radius $r$ and we are taking the limit $r \rightarrow 1^{-}$. So let us parametrize

$$
y=1 \cdot \hat{\Omega}_{3}^{y}, \quad x=r \cdot \hat{\Omega}_{3}^{x}
$$

If $\hat{\Omega}_{3}^{y} \neq \hat{\Omega}_{3}^{x}$ then the contributions between the two points $\left(\hat{\Omega}_{3}^{y}, \hat{\Omega}_{3}^{x}\right)$ and $\left(\hat{\Omega}_{3}^{x}, \hat{\Omega}_{3}^{y}\right)$ cancel in the limit $r \rightarrow 1$. So one might conclude that the integral vanishes, however the previous argument cannot be applied for the point $\hat{\Omega}_{3}^{y}=\hat{\Omega}_{3}^{x}$ where we may have a $\delta$-function-like contribution. To check the contribution to $\mathcal{E}$ from the region where $x \rightarrow y$ we can use the OPE between $\Phi_{i}$ and $\bar{\Phi}_{j}$.

We consider the general form of the OPE between the two scalars

$$
\Phi_{i}(x) \bar{\Phi}_{j}(0)=\sum_{\mathcal{O}} C_{i \bar{j}}^{\mathcal{O}} \frac{1}{|x|^{\Delta_{i}+\Delta_{j}-\Delta_{\mathcal{O}}}} \frac{x^{\mu_{1}} \ldots x^{\mu_{l}}}{|x|^{l}} \mathcal{O}_{\mu_{1} \ldots \mu_{l}}(0)+\text { descendants }
$$


where $l=j+\bar{j}$ is the spin of the operator ${ }^{24} \mathcal{O}$ and $\Delta_{\mathcal{O}}$ its conformal dimension. It turns out that we can have a nontrivial contribution only from an intermediate operator which gives a divergent contribution as $x \rightarrow y$. This happens when

$$
\Delta_{\mathcal{O}}<\Delta_{i}+\Delta_{j}=4
$$

so we have to check for potential contributions from operators (primary or not) of dimension less than four.

Let us start with the contribution from scalars. The lowest lying scalar is the identity operator with $\Delta=0$. In general the conformal dimension of scalar operators obeys $\Delta \geq 1$, except for the identity $\Delta=0$. However in superconformal field theories, if we only consider scalar operators which are uncharged under the $\mathrm{U}(1)_{\mathrm{R}}$ then the unitarity bound becomes $\Delta \geq 2$. So the only candidate scalar operators are the identity and those with $\Delta \geq 2$, as well as their descendants. For vectors the unitarity bound is $\Delta \geq 3$, so we need to consider vectors in the window $3 \leq \Delta<4$. Any descendant of a vector operator satisfies $\Delta \geq 4$ so it cannot contribute. For higher spin $(l \geq 2)$ the unitarity bound for such operators is $\Delta \geq l+2$ so it means $\Delta \geq 4$ and these operators do not contribute.

To summarize we have to consider the identity operator, scalars for $2 \leq \Delta<4$ and vectors with $3 \leq \Delta<4$ as well as their descendants.

\section{C.1 Scalars}

Let us consider the contribution of an intermediate scalar primary operator $\mathcal{O}$ of dimension $\Delta$ to (C.1)

$$
C_{i \bar{j}}^{\mathcal{O}} C_{\mathcal{O} k \bar{l}} \lim _{r \rightarrow 1^{-}} \int_{|y|=1} d \Omega_{3}^{y} \int_{|x|=r} d \Omega_{3}^{x}|x|^{2}|y|^{2}\left(y \cdot \partial_{y}\right)\left(x \cdot \partial_{x}\right)\left(\frac{|y|^{4+\Delta}-|x|^{4+\Delta}}{|x-y|^{4-\Delta}|x|^{2+\Delta}|y|^{2+\Delta}}\right)
$$

We start with the case $\Delta=0$ corresponding to the identity operator. By rotational invariance the integrand depends only on $r$ and the angle $\theta$ between the 4-vectors $x, y$, defined by $x \cdot y=r \cos \theta$. After some algebra we find that the integrand can be written as

$$
\begin{aligned}
|x|^{2}|y|^{2}\left(y \cdot \partial_{y}\right)\left(x \cdot \partial_{x}\right) & \left(\frac{|y|^{4}-|x|^{4}}{|x-y|^{\mid}|x|^{2}|y|^{2}}\right) \\
& =4 \frac{r^{6}+5 r^{4}+5 r^{2}+1-\left(5 r^{4}+2 r^{2}+5\right) r \cos \theta}{\left(r^{2}-2 r \cos \theta+1\right)^{4}}\left(1-r^{2}\right)
\end{aligned}
$$

So the contribution to $\mathcal{E}$ is

$$
(2 \pi)^{3} g_{i \bar{j}} g_{k \bar{l}} \lim _{r \rightarrow 1^{-}} \int_{0}^{\delta} d \theta \sin ^{2} \theta 4 \frac{r^{6}+5 r^{4}+5 r^{2}+1-\left(5 r^{4}+2 r^{2}+5\right) r \cos \theta}{\left(r^{2}-2 r \cos \theta+1\right)^{4}}\left(1-r^{2}\right)
$$

where $\delta$ is a small positive constant which is kept fixed as $r \rightarrow 1$. After some work one can show that the integral is independent of $\delta$ and equal to $2 \pi$, so the contribution from the identity operator is

$$
(2 \pi)^{4} g_{i \bar{j}} g_{k \bar{l}}
$$

\footnotetext{
${ }^{24}$ It is generally true that in the OPE of two scalars we do not have any primary operators of spin $(j, \bar{j})$ with $j \neq \bar{j}$.
} 
where we used the obvious relations $C_{i \bar{j}}^{1}=g_{i \bar{j}}$ and $C_{1 k, \bar{l}}=g_{k \bar{l}}$.

Now let us consider the general case of a scalar primary $\mathcal{O}$ of conformal dimension $\Delta \geq 2$. Its contribution to (C.1) is

$$
C_{i \bar{j}}^{\mathcal{O}} C_{\mathcal{O} k \bar{l}} \lim _{r \rightarrow 1^{-}} \int_{0}^{\delta} d \theta I_{\Delta}(r, \theta)
$$

where the integrand is a function of $r, \theta$ whose precise form is somewhat complicated and not so enlightening to be presented in detail. One can see that if $\theta \neq 0$ then the integrand goes to zero as $r \rightarrow 1$. So we can also choose $\delta=\pi$ to make the integration easier. In that case the $\theta$ integral can be done analytically and we find

$$
\lim _{r \rightarrow 1^{-}} \int_{0}^{\pi} d \theta I_{\Delta}(r, \theta)=0, \quad \Delta>2
$$

and

$$
\int_{0}^{\pi} d \theta I_{\Delta}(r, \theta)=-3(2 \pi)^{4} r^{4}, \quad \Delta=2
$$

So the only potential contribution is from a scalar operator with $\Delta=2, R=0, I=0, j=$ $\bar{j}=0$. This is the special operator $\mathcal{A}$ whose multiplets includes the superconformal currents and stress tensor. We assume it is unique, otherwise there would be two conserved spin-2 currents $^{25}$. The contribution from the operator $\mathcal{A}$ will be

$$
-3(2 \pi)^{4} C_{i \bar{j}}^{\mathcal{A}} C_{\mathcal{A} k \bar{l}}
$$

We now consider the contribution from the first descendant of an operator $\mathcal{O}$ of dimension $\Delta$. In the OPE it appears with the following normalization

$$
\phi_{i}(x) \overline{\phi_{j}}(y) \sim \frac{C_{i \bar{j}}^{\mathcal{O}}}{|x-y|^{4-\Delta}}\left(\mathcal{O}(y)+\frac{1}{2}(x-y)^{\mu} \partial_{\mu} \mathcal{O}(y)+\ldots\right)
$$

Its contribution to the 4-point function $\left\langle\bar{\phi}_{l}(\infty) \Phi_{i}(x) \bar{\Phi}_{j}(y) \phi_{k}(0)\right\rangle$ is

$$
\frac{C_{i \bar{j}}^{\mathcal{O}} C_{\mathcal{O} k \bar{l}}}{2} \frac{1}{|x-y|^{4-\Delta}}(x-y)^{\mu} \partial_{\mu} \frac{1}{|y|^{\Delta}}
$$

Plugging into (C.1) and computing the integral we find that only the case $\Delta=2$ contributes equal to

$$
(2 \pi)^{4} C_{i \bar{j}}^{\mathcal{A}} C_{\mathcal{A} k \bar{l}}
$$

For the second descendants the form of the OPE is

$$
\Phi_{i}(x) \overline{\Phi_{j}}(y) \sim \frac{1}{|x-y|^{4-\Delta}}(x-y)^{\mu}(x-y)^{\nu} \partial_{\mu} \partial_{\nu} \mathcal{O}(y)
$$

\footnotetext{
${ }^{25}$ This assumption is not correct in the free theory where we also have the Konishi scalar with $\Delta=2$, in addition to $\mathcal{A}$. However, when we turn on the coupling the Konishi multiplet gets anomalous dimensions. So we will assume that a generic point on the moduli space of the CFT there is no other scalar with the same quantum numbers as $\mathcal{A}$. It would be interesting to explore this assumption in more detail.
} 
so its contribution to the 4-point function is

$$
\frac{1}{|x-y|^{4-\Delta}}(x-y)^{\mu}(x-y)^{\nu} \partial_{\mu} \partial_{\nu} \frac{1}{|y|^{2 \Delta}}
$$

This does not contribute for any $\Delta \geq 2$. Similarly we find that higher descendants do not contribute.

To summarize, we find that the total contribution to $\mathcal{E}$ from all scalars with $\Delta>0$ and their descendants is the sum of (C.3) and (C.4) or

$$
-2(2 \pi)^{4} C_{i \bar{j}}^{\mathcal{A}} C_{\mathcal{A} k \bar{l}}
$$

Using the results from appendix B we find that this is equal to

$$
\frac{2}{3 c} R g_{i \bar{j}} g_{k \bar{l}}
$$

where $R$ is the $\mathrm{U}(1)_{\mathrm{R}}$ charge of $\phi_{k}$.

\section{C.2 Spin 1}

First we consider conserved currents, so that they have dimension $\Delta=3$. If we want the marginal operator to be neutral under the current (otherwise the symmetry would not be preserved after the marginal deformation and it would be "accidental" and not present at a generic point on the moduli space), then only the $\mathrm{U}(1)_{\mathrm{R}}$ current can appear, since the supercharges carry R-charge and can make the marginal operator neutral even if the chiral primary itself is charged (supercharges are uncharged under the non R-symmetries).

Let us call $R$ the $\mathrm{U}(1)_{\mathrm{R}}$ charge of $\phi_{k}$, while that of $\phi_{i}$ is 4 . According to the analysis of appendix B, we find that the contribution to the 4-point function $\left\langle\bar{\phi}_{l}(\infty) \Phi_{i}(x) \bar{\Phi}_{j}(y) \phi_{k}(0)\right\rangle$ is

$$
\frac{1}{12 c} R g_{i \bar{j}} g_{k \bar{l}} \frac{(x \cdot y)-y^{2}}{|y|^{4}|x-y|^{2}}
$$

After some algebra we conclude that the contribution of the current to (C.1) is

$$
(2 \pi)^{4} g_{i \bar{j}} g_{k i} \frac{R}{12 c}
$$

Similarly we can show that its descendants do not contribute, and also that spin 1 fields of $\Delta>3$ do not contribute.

\section{C.3 Spin $\geq 2$}

A similar analysis shows that no intermediate operators of $l \geq 2$ can contribute to (C.1).

\section{C.4 Final answer for $\mathcal{E}$}

Putting everything together we find that (C.1) is equal to

$$
\mathcal{E}=(2 \pi)^{4} g_{i \bar{j}} g_{k \bar{l}}\left(1-\frac{3 R}{4 c}\right)
$$




\section{Chiral-Antichiral OPE}

Let us consider the OPE of a scalar antichiral primary $\bar{\Phi}_{j}$ of $\Delta=2, R=-4$ with a (for simplicity) scalar chiral primary $\phi_{k}$ of $\Delta>2$ and $R=2 \Delta$. Consider a scalar primary operator $\mathcal{O}$ appearing on the r.h.s. with dimension $\Delta^{\prime}$ and $\mathrm{U}(1)_{\mathrm{R}}$ charge $R^{\prime}$. We have

$$
\bar{\Phi}_{j}(x) \phi_{k}(0) \sim D_{\bar{j} k}^{\mathcal{O}} \frac{\mathcal{O}(0)}{|x|^{2+\Delta-\Delta^{\prime}}}+\ldots
$$

From $\mathrm{U}(1)_{\mathrm{R}}$ charge conservation we have $R^{\prime}=2 \Delta-4$ and unitarity implies $\Delta^{\prime} \geq \frac{R^{\prime}}{2}$ or $\Delta^{\prime} \geq \Delta-2$. We find that the term on the r.h.s. of (D.1) goes like $\frac{1}{|x|^{4}}$ when this inequality is saturated $\Delta^{\prime}=\Delta-2$ which implies that $\mathcal{O}$ is one of the chiral primaries, say $\phi_{n}$. Then it is not difficult to show that

$$
D_{\bar{j} k}^{\mathcal{O}}=g_{k \bar{r}} C_{\bar{j} \bar{p}}^{* \bar{p}} g^{\bar{p} n}
$$

Open Access. This article is distributed under the terms of the Creative Commons Attribution Noncommercial License which permits any noncommercial use, distribution, and reproduction in any medium, provided the original author(s) and source are credited.

\section{References}

[1] N. Seiberg and E. Witten, Monopoles, duality and chiral symmetry breaking in $N=2$ supersymmetric QCD, Nucl. Phys. B 431 (1994) 484 [hep-th/9408099] [SPIRES].

[2] P.C. Argyres, M.R. Plesser and A.D. Shapere, The Coulomb phase of $N=2$ supersymmetric QCD, Phys. Rev. Lett. 75 (1995) 1699 [hep-th/9505100] [SPIRES].

[3] P.C. Argyres and M.R. Douglas, New phenomena in SU(3) supersymmetric gauge theory, Nucl. Phys. B 448 (1995) 93 [hep-th/9505062] [SPIRES].

[4] P.C. Argyres, M. Ronen Plesser, N. Seiberg and E. Witten, New $N=2$ superconformal field theories in four dimensions, Nucl. Phys. B 461 (1996) 71 [hep-th/9511154] [SPIRES].

[5] T. Eguchi, K. Hori, K. Ito and S.-K. Yang, Study of $N=2$ superconformal field theories in 4 dimensions, Nucl. Phys. B 471 (1996) 430 [hep-th/9603002] [SPIRES].

[6] T. Eguchi and K. Hori, $N=2$ superconformal field theories in 4 dimensions and $A-D-E$ classification, hep-th/9607125 [SPIRES].

[7] J.A. Minahan and D. Nemeschansky, An $N=2$ superconformal fixed point with $E_{6}$ global symmetry, Nucl. Phys. B 482 (1996) 142 [hep-th/9608047] [SPIRES].

[8] J.A. Minahan and D. Nemeschansky, Superconformal fixed points with E(n) global symmetry, Nucl. Phys. B 489 (1997) 24 [hep-th/9610076] [SPIRES].

[9] O. Aharony, A. Fayyazuddin and J.M. Maldacena, The large- $N$ limit of $N=2,1$ field theories from three-branes in F-theory, JHEP 07 (1998) 013 [hep-th/9806159] [SPIRES].

[10] P.C. Argyres, M. Crescimanno, A.D. Shapere and J.R. Wittig, Classification of $N=2$ superconformal field theories with two-dimensional Coulomb branches, hep-th/0504070 [SPIRES].

[11] P.C. Argyres and J.R. Wittig, Classification of $N=2$ superconformal field theories with two-dimensional Coulomb branches. II, hep-th/0510226 [SPIRES]. 
[12] P.C. Argyres and J.R. Wittig, Infinite coupling duals of $N=2$ gauge theories and new rank 1 superconformal field theories, JHEP 01 (2008) 074 [arXiv: 0712.2028] [SPIRES].

[13] D. Gaiotto, N=2 dualities, arXiv:0904.2715 [SPIRES].

[14] R. Dijkgraaf, H.L. Verlinde and E.P. Verlinde, Topological strings in $d<1$, Nucl. Phys. B 352 (1991) 59 [SPIRES].

[15] R. Dijkgraaf, H.L. Verlinde and E.P. Verlinde, Notes on topological string theory and 2-D quantum gravity [SPIRES].

[16] E. Witten, On the structure of the topological phase of two-dimensional gravity, Nucl. Phys. B 340 (1990) 281 [SPIRES].

[17] S. Cecotti and C. Vafa, Topological antitopological fusion, Nucl. Phys. B 367 (1991) 359 [SPIRES].

[18] J. de Boer, J. Manschot, K. Papadodimas and E. Verlinde, The chiral ring of AdS3/CFT2 and the attractor mechanism, JHEP 03 (2009) 030 [arXiv:0809.0507] [SPIRES].

[19] K. Papadodimas, New exact results in $4 d$ SCFTs, work in progress.

[20] S. Minwalla, Restrictions imposed by superconformal invariance on quantum field theories, Adv. Theor. Math. Phys. 2 (1998) 781 [hep-th/9712074] [SPIRES].

[21] F.A. Dolan and H. Osborn, On short and semi-short representations for four dimensional superconformal symmetry, Ann. Phys. 307 (2003) 41 [hep-th/0209056] [SPIRES].

[22] W. Lerche, C. Vafa and N.P. Warner, Chiral Rings in $N=2$ Superconformal Theories, Nucl. Phys. B 324 (1989) 427 [SPIRES].

[23] K. Ranganathan, Nearby CFTs in the operator formalism: The Role of a connection, Nucl. Phys. B 408 (1993) 180 [hep-th/9210090] [SPIRES].

[24] H. Sonoda, Composite operators in QCD, Nucl. Phys. B 383 (1992) 173 [hep-th/9205085] [SPIRES].

[25] H. Sonoda, Connection on the theory space, hep-th/9306119 [SPIRES].

[26] N. Seiberg, Observations on the moduli space of superconformal field theories, Nucl. Phys. B 303 (1988) 286 [SPIRES].

[27] D. Kutasov, Geometry on the space of conformal field theories and contact terms, Phys. Lett. B 220 (1989) 153 [SPIRES].

[28] G. Bonelli and M. Matone, Nonperturbative relations in $N=2$ SUSY Yang-Mills and WDVV equation, Phys. Rev. Lett. 77 (1996) 4712 [hep-th/9605090] [SPIRES].

[29] A. Marshakov, A. Mironov and A. Morozov, WDVV-like equations in $N=2$ SUSY Yang-Mills theory, Phys. Lett. B 389 (1996) 43 [hep-th/9607109] [SPIRES].

[30] A. Marshakov, A. Mironov and A. Morozov, WDVV equations from algebra of forms, Mod. Phys. Lett. A 12 (1997) 773 [hep-th/9701014] [SPIRES].

[31] A. Marshakov, A. Mironov and A. Morozov, More evidence for the WDVV equations in $N=2$ SUSY Yang-Mills theories, Int. J. Mod. Phys. A 15 (2000) 1157 [hep-th/9701123] [SPIRES].

[32] B. de Wit and A. Van Proeyen, Potentials and symmetries of general gauged $N=2$ supergravity: Yang-Mills models, Nucl. Phys. B 245 (1984) 89 [SPIRES]. 
[33] A. Strominger, Special geometry, Commun. Math. Phys. 133 (1990) 163 [SPIRES].

[34] V. Periwal and A. Strominger, Kähler geometry of the space of $N=2$ superconformal field theories, Phys. Lett. B 235 (1990) 261 [SPIRES].

[35] M. Bershadsky, S. Cecotti, H. Ooguri and C. Vafa, Kodaira-Spencer theory of gravity and exact results for quantum string amplitudes, Commun. Math. Phys. 165 (1994) 311 [hep-th/9309140] [SPIRES].

[36] B.R. Greene, D.R. Morrison and M.R. Plesser, Mirror manifolds in higher dimension, Commun. Math. Phys. 173 (1995) 559 [hep-th/9402119] [SPIRES].

[37] A. Petkou and K. Skenderis, A non-renormalization theorem for conformal anomalies, Nucl. Phys. B 561 (1999) 100 [hep-th/9906030] [SPIRES].

[38] A. Basu, M.B. Green and S. Sethi, Some systematics of the coupling constant dependence of $N=4$ Yang-Mills, JHEP 09 (2004) 045 [hep-th/0406231] [SPIRES].

[39] S. Cecotti, N=2 Landau-Ginzburg versus Calabi-Yau $\sigma$-models: Nonperturbative aspects, Int. J. Mod. Phys. A 6 (1991) 1749 [SPIRES].

[40] S. Helgason, Differential geometry and symmetric spaces, Academic Press (196).

[41] P.C. Argyres and A. Buchel, The nonperturbative gauge coupling of $N=2$ supersymmetric theories, Phys. Lett. B 442 (1998) 180 [hep-th/9806234] [SPIRES].

[42] N. Seiberg and E. Witten, Monopole condensation, and confinement in $N=2$ supersymmetric Yang-Mills theory, Nucl. Phys. B 426 (1994) 19 [Erratum ibid. B 430 (1994) 485] [hep-th/9407087] [SPIRES].

[43] E. Witten, Topological quantum field Theory, Commun. Math. Phys. 117 (1988) 353 [SPIRES].

[44] V. Pestun, Localization of gauge theory on a four-sphere and supersymmetric Wilson loops, arXiv:0712.2824 [SPIRES].

[45] A.D. Shapere and Y. Tachikawa, Central charges of $N=2$ superconformal field theories in four dimensions, JHEP 09 (2008) 109 [arXiv:0804.1957] [SPIRES].

[46] P.C. Argyres and N. Seiberg, S-duality in $N=2$ supersymmetric gauge theories, JHEP 12 (2007) 088 [arXiv:0711.0054] [SPIRES].

[47] D. Gaiotto and J. Maldacena, The gravity duals of $N=2$ superconformal field theories, arXiv: 0904.4466 [SPIRES].

[48] L.F. Alday, D. Gaiotto and Y. Tachikawa, Liouville Correlation Functions from Four-dimensional Gauge Theories, Lett. Math. Phys. 91 (2010) 167 [arXiv:0906.3219] [SPIRES].

[49] L.F. Alday, D. Gaiotto, S. Gukov, Y. Tachikawa and H. Verlinde, Loop and surface operators in $N=2$ gauge theory and Liouville modular geometry, JHEP 01 (2010) 113 [arXiv: 0909.0945] [SPIRES].

[50] K.A. Intriligator, R.G. Leigh and N. Seiberg, Exact superpotentials in four-dimensions, Phys. Rev. D 50 (1994) 1092 [hep-th/9403198] [SPIRES].

[51] Y. Tachikawa, Five-dimensional supergravity dual of a-maximization, Nucl. Phys. B 733 (2006) 188 [hep-th/0507057] [SPIRES].

[52] K.A. Intriligator and B. Wecht, The exact superconformal R-symmetry maximizes a, Nucl. Phys. B 667 (2003) 183 [hep-th/0304128] [SPIRES]. 
[53] J. Kinney, J.M. Maldacena, S. Minwalla and S. Raju, An index for 4 dimensional super conformal theories, Commun. Math. Phys. 275 (2007) 209 [hep-th/0510251] [SPIRES].

[54] M. Kontsevich and Y. Soibelman, Stability structures, motivic Donaldson-Thomas invariants and cluster transformations, arXiv:0811.2435.

[55] D. Gaiotto, G.W. Moore and A. Neitzke, Four-dimensional wall-crossing via three-dimensional field theory, arXiv:0807.4723 [SPIRES].

[56] S. Cecotti and C. Vafa, BPS wall crossing and topological strings, arXiv:0910.2615 [SPIRES].

[57] M.V. Berry, Quantal phase factors accompanying adiabatic changes, Proc. Roy. Soc. Lond. A 392 (1984) 45 [SPIRES].

[58] F. Wilczek and A. Zee, Appearance of gauge structure in simple dynamical systems, Phys. Rev. Lett. 52 (1984) 2111 [SPIRES].

[59] J. de Boer, K. Papadodimas and E. Verlinde, Black hole berry phase, Phys. Rev. Lett. 103 (2009) 131301 [arXiv:0809.5062] [SPIRES].

[60] C. Pedder, J. Sonner and D. Tong, The geometric phase and gravitational precession of D-branes, Phys. Rev. D 76 (2007) 126014 [arXiv:0709.2136] [SPIRES].

[61] C. Pedder, J. Sonner and D. Tong, The geometric phase in supersymmetric quantum mechanics, Phys. Rev. D 77 (2008) 025009 [arXiv:0709.0731] [SPIRES].

[62] C. Pedder, J. Sonner and D. Tong, The berry phase of D0-branes, JHEP 03 (2008) 065 [arXiv:0801.1813] [SPIRES].

[63] J. Sonner and D. Tong, Berry phase and supersymmetry, JHEP 01 (2009) 063 [arXiv:0810.1280] [SPIRES].

[64] L.J. Dixon, V. Kaplunovsky and J. Louis, On effective field theories describing (2,2) vacua of the heterotic string, Nucl. Phys. B 329 (1990) 27 [SPIRES].

[65] M.F. Sohnius, The multiplet of currents for $N=2$ extended supersymmetry, Phys. Lett. B 81 (1979) 8 [SPIRES].

[66] F.A. Dolan and H. Osborn, Superconformal symmetry, correlation functions and the operator product expansion, Nucl. Phys. B 629 (2002) 3 [hep-th/0112251] [SPIRES].

[67] S.M. Kuzenko and S. Theisen, Correlation functions of conserved currents in $N=2$ superconformal theory, Class. Quant. Grav. 17 (2000) 665 [hep-th/9907107] [SPIRES]. 